\title{
Modeling and forecasting time series of precious metals: a new approach to multifractal data
}

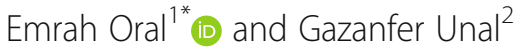

\author{
*Correspondence: emrahoral@ \\ gmail.com \\ ${ }^{1}$ Faculty of Economy and \\ Administrative Sciences, Istanbul \\ Aydin University, Istanbul, Turkey \\ Full list of author information is \\ available at the end of the article
}

\begin{abstract}
We introduce a novel approach to multifractal data in order to achieve transcended modeling and forecasting performances by extracting time series out of local Hurst exponent calculations at a specified scale. First, the long range and co-movement dependencies of the time series are scrutinized on time-frequency space using multiple wavelet coherence analysis. Then, the multifractal behaviors of the series are verified by multifractal de-trended fluctuation analysis and its local Hurst exponents are calculated. Additionally, root mean squares of residuals at the specified scale are procured from an intermediate step during local Hurst exponent calculations. These internally calculated series have been used to estimate the process with vector autoregressive fractionally integrated moving average (VARFIMA) model and forecasted accordingly. In our study, the daily prices of gold, silver and platinum are used for assessment. The results have shown that all metals do behave in phase movement on long term periods and possess multifractal features. Furthermore, the intermediate time series obtained during local Hurst exponent calculations still appertain the co-movement as well as multifractal characteristics of the raw data and may be successfully re-scaled, modeled and forecasted by using VARFIMA model. Conclusively, VARFIMA model have notably surpassed its univariate counterpart (ARFIMA) in all efficacious trials while re-emphasizing the importance of comovement procurement in modeling. Our study's novelty lies in using a multifractal de-trended fluctuation analysis, along with multiple wavelet coherence analysis, for forecasting purposes to an extent not seen before. The results will be of particular significance to finance researchers and practitioners.
\end{abstract}

Keywords: Continuous wavelet transform, Multiple wavelet coherence, Multifractal de-trended fluctuation analysis, Vector autoregressive fractionally integrated moving average, Forecast

\section{Introduction}

Multifractal structure analysis has become more and more popular in financial studies. It is referred as one of the strong and dynamic techniques due to its ability to detect multifractal behavior in non-stationary time series. Especially, local Hurst exponents help point out the discontinuities in the financial time series. Hence, any asymmetric or inconsistent behavior in the time series, such as the failure of any economic system, can be captured. These irregularities are the main reason for the fat tail observations. Local Hurst 
exponents demonstrate that these irregular behaviors may be organized to be used in various models/methods.

Mandelbrot and Ness has laid the foundations of multifractal analysis by introducing fractional Brownian motions, fractional noises and its applications (Mandelbrot \& Van Ness, 1968). Later, multifractal de-trended fluctuation analysis (MF-DFA) has been proposed as an alternative method in analyzing financial time series by (Kantelhardt et al., 2002). Heretofore, there have been many researchers using this method in their analysis. Zhang et al. have investigated asymmetric multiscale multifractal analysis of wind speed signals (Zhang et al., 2017). Multifractal and wavelet analysis of epileptic seizures have been studied by (Dick \& Mochovikova, 2011). An in-depth analysis of stocks of the company GE was performed by Thomson and Wilson by contrasting the results with those obtained using multifractal de-trended fluctuation analysis and using conventional time series models (Thompson \& Wilson, 2014). Benbachir and Alaoui employed the MF-DFA method in order to explore the multifractal properties of the Moroccan Dirham compared with the US Dollars (Benbachir \& Alaoui, 2011). Zhu and Zhang studies the multifractal property of Chinese stock market in the CSI 800 index based on MF-DFA approach (Zhu \& Zhang, 2017) and concluded that the shape and width of multifractal spectrum are dependent on the weighing order and Hurst exponents can account for the market crash. (Sensoy, 2013) studies the time-varying efficiency of 15 Middle East and North African (MENA) stock markets by generalized Hurst exponent analysis of daily data with a rolling window technique and concludes that all MENA stock markets exhibits different long range dependence. In other study, Sensoy and Tabak proposes an alternative index to model time-varying inefficiency in stock markets using generalized Hurst exponents calculations (Sensoy \& Tabak, 2015). (Tiwari et al., 2017) in 2016 challenges efficient hypothesis using the generalized Hurst exponent and MF-DFA methods. In our study, we will show that obtaining coherent time series lead to more accurate forecasting results because not only the long-run effects but also the short and long term dynamics can be taken into considerations simultaneously.

The wavelet analysis is also one of the methods in scrutinizing the time series. Unlike Fourier transform, as Burrus et al. indicated, wavelet analysis does not need stationarity (Burrus et al., 1998) and is able to look deep into the frequency information of the series at different scales without losing time information (Reboredo \& Rivera-Castro, 2014) which helps eliminate the weaknesses in Fourier transform (Gülerce \& Ünal, 2016). Gencay's study (Gencay, 2002) has become a pioneer work for many researchers using wavelet tools to analyze financial time series in many studies such as (Aguiar-Conraria \& Soares, 2012; Aguiar-Conraria \& Soares, 2013), (Barunik et al. 2013), and (McCarthy \& Orlov, 2012). Multi-scale analysis is accepted as one of the main applications of wavelet methods in finance and economics (Haven, 2012).

There have been many investigations looking into the relation of precious metals using these types of tools. Kucher and McCoskey indicated that the long-run relationships between precious metal prices are strongly influenced by economic conditions using a vector error correction model (Kucher \& McCoskey, 2017). A flexible modification of the dynamic conditional correlation model that accounts for asymmetry and long memory in variance is applied on precious metals by (Klein, 2017). He et al. uses wavelet analysis and autoregressive moving average model with higher accuracy to forecast prices of precious metals (He et al., 2017). Das et al. suggest intense and positive co-movement in Asian gold spot markets after the Asian financial crises of 1997 at all frequencies (Das et al., 2017). To the best of 
our knowledge, multifractal de-trended fluctuation analysis along with multiple wavelet coherence analysis of precious metals has not been studied to this extent heretofore.

In this paper, we will use a software package for wavelet coherence analysis provided by (Torrence \& Compo, 1998) and (Grinsted et al., 2004) and a Matlab tool for multifractal de-trended fluctuation analysis developed by (Ihlen, 2012). The former will be used to analyze inter relations, co-movement dependencies, frequent and consistent signals in multiple financial time series of precious metals (gold, silver and platinum). The latter will be used to confirm their multifractal behavior, calculate local Hurst exponents and obtain the inter-calculated fractal function time series at specific scale determined.

Multiple wavelet coherence will be used to determine the specific time period and scale that possesses common long range dependence out of the time series. With the help of Matlab tool, the multifractal behavior of these time series will be validated and a new series out of local Hurst exponent calculations will be obtained. These new series will be modeled using multivariate methods and forecasted accordingly. The first multivariate model has been introduced by Quenouille in 1957 (Akaike, 1974) and later improved by Akaike (Dunsmuir \& Hannan, 1976), Dunsmuir and Hannan (Hannan, 1981) and Hannan (Oral \& Unal, 2017a) in order. Multivariate models are a dynamic system of equations that examine the impacts of fluctuations (shocks) or correlations (interactions) between financial variables (Oral \& Unal, 2017a). Multivariate modeling is because of the fact that more information out of multiple highly correlated data can be used and low mean-squared errors compared to univariate models may be obtained (Oral \& Unal, 2017b). Moreover, multifractal nature of our data urges us to employ vector autoregressive fractionally integrated moving average method.

Gold is treated as hedging instrument against inflation and exchange rates (Hammoudeh et al., 2010) and, in many papers, reported as an indicator of inflation (Ranson \& Wainright, 2005). Likewise, Mahdavi and Zhou points that commodity prices respond to new information faster than any consumer price (Mahdavi \& Zhou, 1997). Therefore, results obtained from a successful forecasting of gold with smaller error bands may help and support both finance researchers as well as many different players in financial world such as monetary policymakers, hedge fund managers, portfolio managers, centrals banks and investors while making investment decisions. As far as we know there is no general method about forecasting of data possessing multifractal nature and we believe our paper will serve well during these type of decision-making processes.

The rest of the paper is organized as follows: Section 2 covers the data and methodology used in this paper. The main equation of continuous wavelet transform and multiple wavelet coherence, basics of multifractal de-trended fluctuations analysis and vector autoregressive fractionally integrated moving average model will be included as a summary review. In section 3, multiple wavelet coherence will be utilized to detect the highly correlated time periods and frequencies. After the multifractal characteristics of the series are verified, a new series of fractional function will be obtained out of local Hurst exponent calculations at the specified scale. Section 4 will compare and discuss the forecasting results of both multivariate and univariate models. Section 5 will be ended with the discussion of the results. In Appendix 1 will display two forecasting results of each metal couple along with the plot of the data set extracted from the local Hurst exponents' calculations at the specified period. 


\section{Data \& Methodology}

Data

The daily prices of gold, silver and platinum (Fig. 1) is acquired from Yahoo Finances! starting from 1st of July, 2011 to 30th of November, 2016 which is composed of approximately 2000 daily data.

As can be seen from Table 1, silver has the highest mean and standard deviation, thus there is higher fluctuation in the prices of silver with respect to other metals. One thing that draws one's attention is the skewness value of platinum. The negative skewness or negatively skewed means frequent small gains and a few extreme losses. This could indicate platinum a safer investment with respect to gold and silver during economic crisis. On the other hand, positive skewness or positively skewed means frequent small losses but a few extreme gains which is greater for silver due to its higher volatility that we pointed.

When we look at the traditional correlation calculation results of these metals as shown in Table 2, we realize that there is higher correlation with gold and silver with respect to platinum. Additionally, platinum seems to move with silver more than with gold. It is surprising to see $85 \%$ correlation with silver and platinum even though the volatility of silver prices is expected to be higher.

In this paper, we will look deep into co-movement of metal prices in time and frequency space by using multiple wavelet coherence. Once highly correlated time interval and frequency is determined, the multifractal behavior of the real series will be validated. A new time series of fluctuation function at the specified scale will be obtained out of its local Hurst exponents calculations. Finally, we will compare and discuss the performance of modeling and forecasting using these series with the help of univariate and multivariate models.

\section{Methodology}

\section{Continuous wavelet transform (CWT) and multiple wavelet coherence}

An application of wavelet analysis in finance and economics were first presented by Gencay in 2002 (Gencay, 2002). (Aguiar-Conraria \& Soares, 2012; Aguiar-Conraria \&

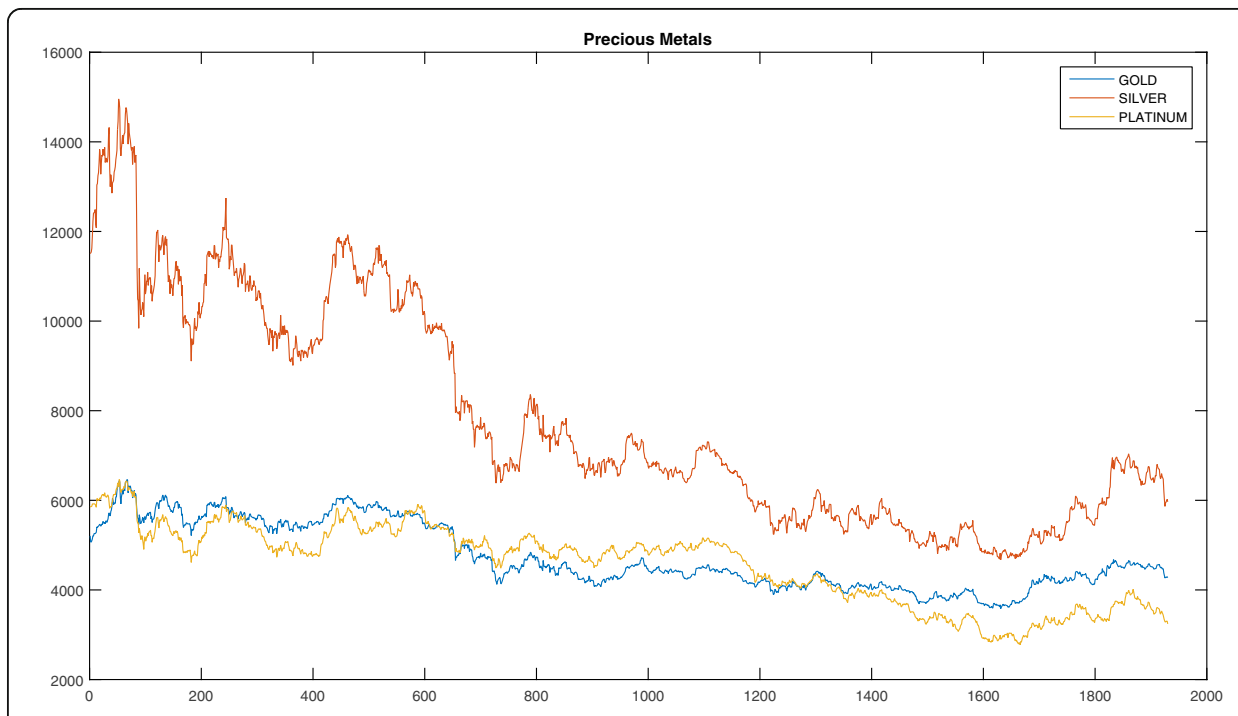

Fig. 1 The daily prices of gold, silver and platinum starting from July, 2011 to November, 2016 
Table 1 Daily Data of Precious Metals from July 2011 to November 2016

\begin{tabular}{llllll}
\hline & Mean & Median & St. Dev. & Kurtosis & Skewness \\
\hline Gold & 4736.03 & 4478.74 & 736.49 & 1.8535 & 0.4855 \\
Silver & 7805.07 & 6845.28 & 2478.29 & 2.4046 & 0.7307 \\
Platinum & 4574.81 & 4843.27 & 872.87 & 2.0483 & -0.2758 \\
\hline
\end{tabular}

Soares, 2013), (Barunik et al., 2013), (Yilmaz \& Unal, 2016) and Oral and Unal (Quenouille, 1957; Oral \& Unal, 2017b) are some of the many researchers who have utilized these tools in their studies. There are many ways how to sort the types of the wavelet transforms. The division based on the wavelet orthogonality defines two methods, discrete wavelet transform (DWT) for orthogonal wavelets and continuous wavelet transform (CWT) for non-orthogonal wavelets. Nevertheless, CWT performs better to see the signal frequencies evolution during the duration of the signal and compare the spectrum with other signals spectra, thus is used more for feature extraction purposes (Grinsted et al., 2004) to see the results in a simpler form. CWT follows the following form in Eq. (1),

$$
C W T_{x}^{\psi}(s, \tau)=\langle X, \rightarrow \psi(s, \tau)\rangle=\frac{1}{\sqrt{s}} \int_{-\infty}^{+\infty} X(t) \psi^{*}\left(\frac{t-\tau}{s}\right) d t
$$

Where the transforming function, also known as the mother wavelet, is " $\psi$ ", the translation parameter is " $\tau$ " and the scale parameter is " $\mathrm{s}$ ". The translation parameter is the time information in the transform domain and the scale parameter is the frequency of the corresponding information.

Wavelet coherence may be evaluated not only in two dimensional data sets but also in multi-dimensional sets of data for the same time and frequency space. Thus, multivariate models where three dimensional multiple wavelet coherence is possible as shown in the following Eq. (2),

$$
\begin{aligned}
& C^{d}=1-R_{23}^{2}-R_{12}^{2}-R_{13}^{2}+\rho_{12} \rho_{23} \rho_{31}+\rho_{13} \rho_{21} \rho_{32} \\
& C^{d}=1-R_{23}^{2}-R_{12}^{2}-R_{13}^{2}+2 R\left(\rho_{12} \rho_{23} \rho_{31}\right) \\
& C^{d}=1-R_{23}^{2}-R_{12}^{2}-R_{13}^{2}+2 R\left(\rho_{12} \rho_{23} \rho_{13}^{*}\right),
\end{aligned}
$$

Where $\mathrm{C}$ denotes complex coherence, $\rho_{i j}$ is the correlation factor and $\mathrm{R}^{2} \mathrm{ij}$ is squared multiple wavelet coherencies.

\section{Multifractal De-trended fluctuation analysis (MF-DFA)}

Multifractal de-trended fluctuation analysis (MF-DFA) may be used to analyze financial time series demonstrating volatility clustering or many different forms of irregular behavior. It is in the nature of all financial time series data to have fractal structures and behavior. As Ihlen pointed, the deviations in fractal structure within time periods with

Table 2 Correlation of gold, silver and platinum

\begin{tabular}{lll}
\hline & Gold & Silver \\
\hline Silver & 0.718556 & \\
Platinum & 0.54741 & 0.8564173 \\
\hline
\end{tabular}


large and small fluctuations may be identified by multifractal analysis (Ihlen, 2012). The main advantage of the method is to be able to detect multifractal behavior in non-stationary time series. That is why it is proposed as a robust and powerful technique by (Kantelhardt et al., 2002). The aim of the MF-DFA is to find the spectrum of singularities both for stationary as well as non-stationary time series. It is assumed that $\mathrm{X}(\mathrm{t})$ is self-similar time series with Hurst exponent $\mathrm{H} \geq 0$. For any $\mathrm{c}>0$, we have

$$
X(c t)=c^{H} X(c t) .
$$

According to Eq. (3), when $\mathrm{H}$ is greater than 0.5 , the time series data possesses persistent behavior with long range dependence. This persistency indicates practicably the same sign for the next non-overlapping time segment in line. If $\mathrm{H}$ is less than 0.5 , the time series data will exhibit anti-persistent behavior with short range dependence. This, however, anti-persistent behavior would mean that an increase (decrease) in the process is most likely to be followed by decrease (increase) in the next time segment.

Assume that $\mathrm{X}(\mathrm{t})$ is a time series of length $\mathrm{N}$ with mean of $\bar{x}$. Firstly, the accumulated profile $\mathrm{y}(\mathrm{i})$ of the time series $\mathrm{X}(\mathrm{t})$ for $\mathrm{i}=1, \ldots, \mathrm{N}$ is determined.

$$
y(i)=\sum_{i=1}^{N}[X(i)-\bar{x}] .
$$

Firstly, a non-overlapping segment of equal length scale $\mathrm{s}$ is formed. Then the profile $y(i)$ is divided to the number $N_{s}=\operatorname{int}(N / s)$ rounding to the nearest integer, int(). The same procedure is repeated starting from the end of the profile in order to include a short part of the end of the profile, doubling the number of segments, $2 \mathrm{~N}_{\mathrm{s}}$.

Each segment is fitted by a polynomial providing a local trend to the data and the variances are calculated by two formulas in terms of segment $\mathrm{v}$. For each segment $\mathrm{v}=$ $1, \ldots, \mathrm{N}_{\mathrm{s}}$ :

$$
F^{s}(v, s)=\frac{1}{s} \sum_{i=1}^{s}\left[y((v-1) s+i)-p_{v}^{n}(i)\right]^{2} .
$$

And for each segment from the end of the profile $\mathrm{v}=\mathrm{N}_{\mathrm{s}}+1, \ldots, 2 \mathrm{~N}_{\mathrm{s}}$ :

$$
F^{s}(v, s)=\frac{1}{s} \sum_{i=1}^{s}\left[y\left(\left(N-v-N_{s}\right) s+i\right)-p_{v}^{n}(i)\right]^{2},
$$

$p_{v}^{n}(i)$ is the $\mathrm{n}^{\text {th }}$ order fitting polynomial in the segment order, v. For $n>3$, any order of polynomials can be used, linear, quadratic, cubic or higher. Next, $\mathrm{q}^{\text {th }}$ order fluctuation functions will be obtained by averaging the variance over all segments. This calculation is shown in Eq. (7) as following, for $\mathrm{q} \neq 0$ :

$$
F_{q}(s)=\left[\frac{1}{2 N_{s}} \sum_{v=1}^{2 N_{s}}\left[F^{2}(v, s)\right]^{q / 2}\right]^{1 / q} .
$$

And if $\mathrm{q}=0$, the equation is evaluated as in Eq. (8)

$$
F_{0}(s)=\exp \left[\frac{1}{2 N_{s}} \sum_{v=1}^{2 N_{s}} \ln \left[F^{2}(v, s)\right]\right]
$$

Resolving the behavior of the q-dependent fluctuations $F_{q}(s)$ is the main goal of MF-DFA while taking into consideration the time scale $\mathrm{s}$ and various values of $\mathrm{q}$. The Eq. 5 thru 8 should be run in loop for various values of time scales s. Hence, the 
multi-scaling behavior of the fluctuation functions $F_{q}(s)$ may be analyzed. In order to do that, the slope of $\log$-log plots of $F_{q}(s)$ with respect to $\mathrm{s}$ for different values of q (such as $-3,-2,-1,0,1,2,3$ ) should be estimated. The fluctuation function $F_{q}(s)$ will demonstrate the following power-law scaling behavior as shown in Eq. (9) depending on the analysis of long-range power-law correlation as fractal proprieties,

$$
F_{q}(s) \sim s^{h(q)} .
$$

For different values of $\mathrm{q}, h(q)$ is regressed on the time series $F_{q}(s)$. The time series is called monofractal, when a constant value for $h(q)$ for all values of q is true. Conversely, the time series is said to be multifractal, when $h(q)$ is a steadily decreasing function of q. The Hurst exponents of $h(q)$ represent the scaling properties of small (large) fluctuations when the values of $\mathrm{q}$ are negative (positive). Ihlen pointed that the size of local Hurst exponents in the periods of the multifractal time series with local fluctuation of different magnitudes determines the structure of the local fluctuations (Ihlen, 2012). Small (large) local Hurst exponents mean large (small) noise like (random walk like) structure of local fluctuations. This is identical with the generalized Hurst exponents for negative and positive values of $\mathrm{q}$, respectively. Regardless, the structural changes within time series are caught instantly with the local Hurst exponents and it is the major advantage compared to generalized Hurst exponent.

\section{Vector autoregressive fractionally integrated moving average model (VARFIMA)}

The efficiency of multivariate time series analysis may be increased by understanding the importance of synchronization of multiple time series. Tsay stated that the long-run effects as well as the short and long term dynamics characterized by the AR, MA and the fractional differencing parameters may be simultaneously addressed by vector autoregressive fractionally integrated moving average (VARFIMA) model (Tsay, 2013). (Sowell, 1989), (Durr et al., 1997), (Dueker \& Startz, 1998), (Box-Steiensmeier \& Tomlinson, 2000), (Clarke \& Lebo, 2003) have all used these models in their time series observations. Primarily, the ARFIMA (p, d, q) process can be described by the difference equation in (10)

$$
\left(1-a_{1} L_{\left|t_{n}-1\right|}-\ldots-a_{p} L_{\left|t_{n}-p\right|}\right)(1-L)^{d} y(t)=\left(1+b_{1} L_{\left|t_{n}-1\right|}+\ldots+b_{p} L_{\left|t_{n}-p\right|}\right) e(t),
$$

where $y(t)$ is the state output, $e(t)$ is the white noise input, and $L$ is the shift operator. $a_{i}$, and $b_{j}$ are real coefficient matrices of $\mathrm{n}$ by $\mathrm{n}$ dimensions, $d$ is real integrating parameter between -0.5 and +0.5 . The parameter $d$ is also called memory parameter because it regulates the long memory property of the series.

The vector ARFIMA process has transfer matrix $t\left(L^{-1}\right)$ where $t(L)$ function can be defined as (11)

$$
t(L)=\left(\left(I_{n}-a_{1} L-\ldots-a_{p} L^{p}\right) \cdot\left(l_{n}-L\right)^{d}\right)^{-1}\left(I_{n}+b_{1} L+\ldots+b_{q} L^{q}\right),
$$

where $I_{n}$ is the identity matrix with dimensions $\mathrm{n}$ by $\mathrm{n}$. Equation (11) can now be written in summary notation as

$$
A(L)(I-L)^{d} y(t)=M(L) \varepsilon(t),
$$


where $L$ is the lag operator and $\mathrm{A}(\mathrm{L})=\left(A_{0}-A_{1} L-\ldots-A_{p} L^{p}\right) .\left(A_{0}-L\right)^{d}$ and $\mathrm{B}(\mathrm{L})=$ $B_{0}+B_{1} L+\ldots+B_{q} L^{q}$. Equation (12) can be written as follows:

$$
y(t)=B(L)\left\{A^{-1}(L)(I-L)^{-d} \varepsilon(t)\right\}=B(L) \xi(t) .
$$

This means a two-step of calculation for $\xi(t)$ first and then for $y(t)$.

From here, we see that VARFIMA model can be reduced in a straightforward way to a set of $\mathrm{n}$ interrelated ARFIMA models. Hence, when we rewrite Eq. (12), we get

$$
y(t)=B(L) \frac{1}{|A(L)|(I-L)^{d}} A^{*}(L) \varepsilon(t)
$$

where $|A(L)|$ is the scalar valued determinant of $\mathrm{A}(L)$ and $A^{\prime \prime}(L)$ is the adjoint matrix. The process becomes

$$
|A(L)|(I-L)^{d} y(t)=B(L) A^{*}(L) \varepsilon(t),
$$

where a system of $\mathrm{n}$-dimensional ARFIMA process is defined with the common lag operator, $L$ and co-integration fraction, $d$.

It is important to underline that forecasting is an overwhelming task. Whilst a statistically sensible model may be obtained, it may still endure severe problems in forecasting. Hence in this paper, the validity of the model is determined by judging the performance of the forecasting results. A stochastic process vector ARFIMA (p, d, q) is already involved in our approach in modeling phase. Once we obtain statistically admissible model parameters ( $\mathrm{p}, \mathrm{d}$, q), the standard best linear predictor of the program is used. The program automatically determines the best option by running three different methods, "Covariance" (exact covariance), "AR" (approximate with a large0order AR process) or "Kalman" (Kalman filter) method.

\section{Wavelet coherence and local Hurst exponents}

Wavelet coherence displays the time series data in two dimensional axes of frequency and time space as shown in Fig. 2. The time information is displayed on $\mathrm{x}$ axis and the frequency information on y axis. The frequency information is also represented as period or scale. However, they are inversely proportional where high frequency means low scale or vice versa. The column on the right indicates the correlation scale. The yellow colors in the figure means higher correlation and the blue colors mean no correlation during the specific time and frequency interval. The power of correlation is one at its highest point and zero where there is no correlation. The black lines represent the statistical significance at $5 \%$ level against red noise. Since the length of the wavelet may exceed the length of the data on the edges, the discontinuities in the wavelet function is displayed in the faded area (Yilmaz \& Unal, 2016). The faded area falls onto the outside of the cone of influence (COI).

Three dimensional multiple wavelet coherence would bring out the effect of two factors together on the commodity itself. As we look at the multiple wavelet coherence of silver with gold and platinum in Fig. 3, it shows a strong relation almost at all times and frequencies. There is a weakening relationship at around 32-day period from time to time. All metals are displaying very strong relation along 256-day period at all times. It was also possible to point out that platinum has the lowest amount of coherence 


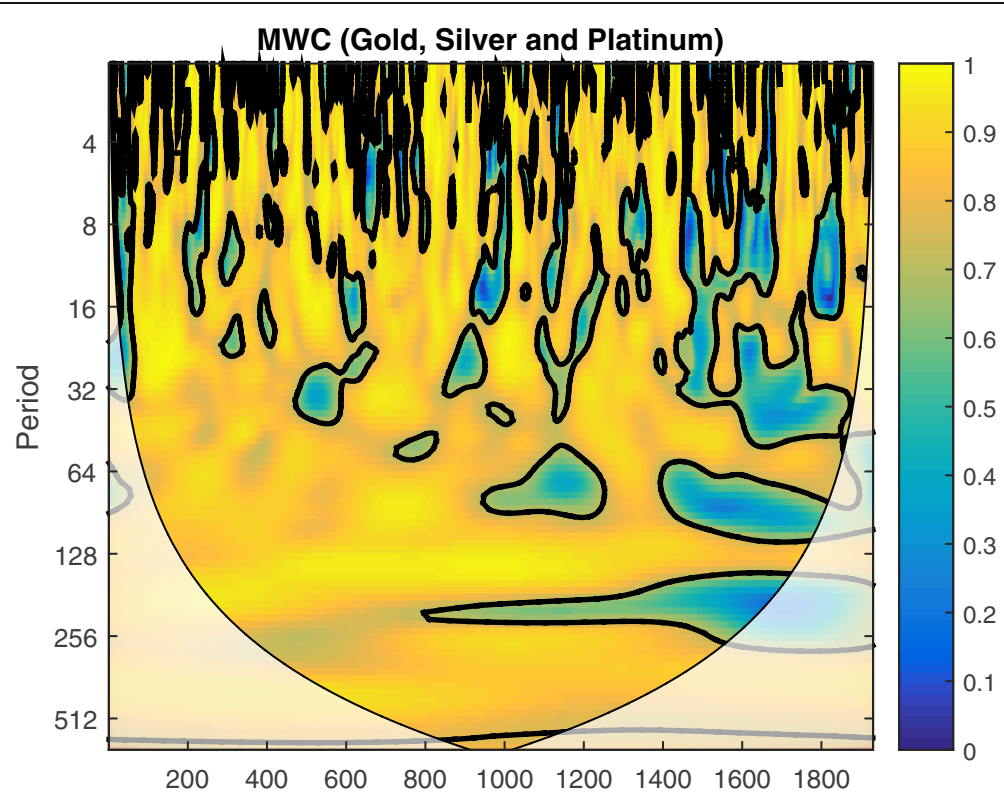

Fig. 2 Multiple wavelet coherence of gold with silver and platinum

with other metals. The traditional correlation calculations have demonstrated themselves in much detail with the wavelet coherence diagrams.

It is seen that there are some of the areas colored blue considering long range dependency. The blue areas indicate low interrelation between the metals at the specific time and frequency. These areas are found more in platinum diagram (Fig. 4) but less in silver diagram (Fig. 3). This may be because of reaching equilibrium in prices at different rates during shocks or stresses. Kucher and McCoskey find in their study that the error correction mechanisms between precious metal prices seem to be asymmetric (Kucher \& McCoskey, 2017). This also aligns with our observations and explains some of the weak long run (low frequency / higher periods) relationship over certain periods of time. The continuous

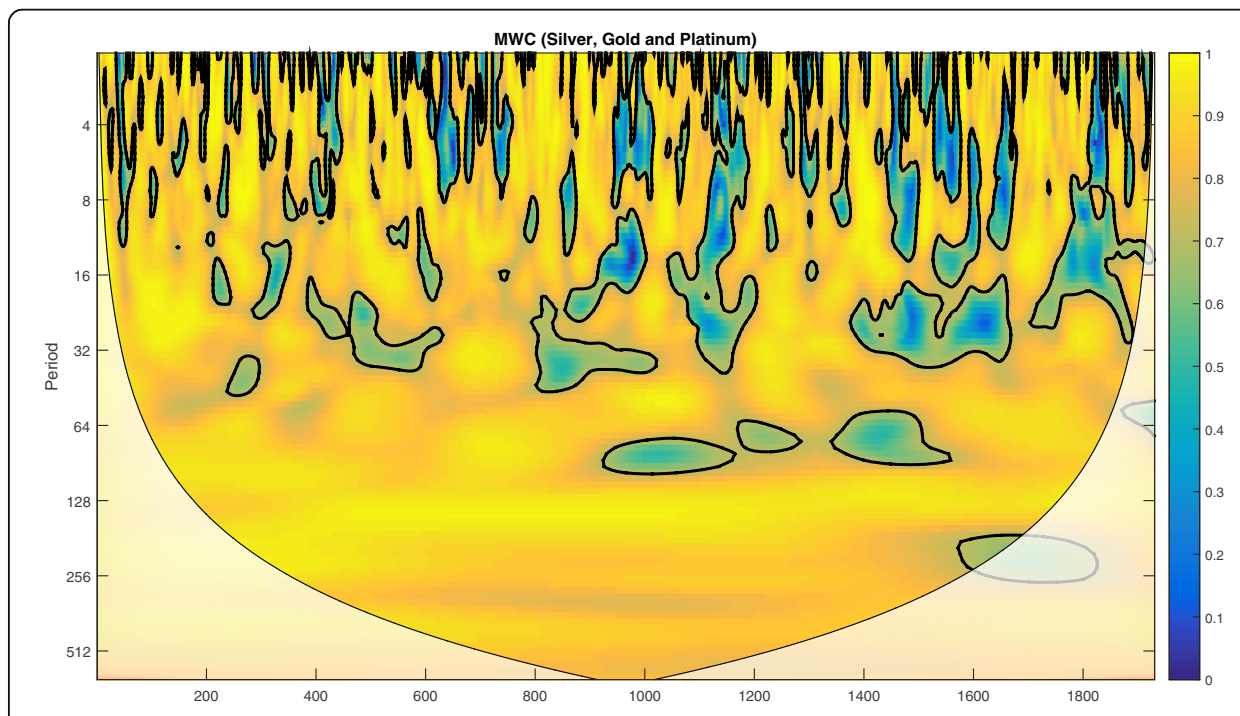

Fig. 3 Multiple wavelet coherence of silver with gold and platinum 


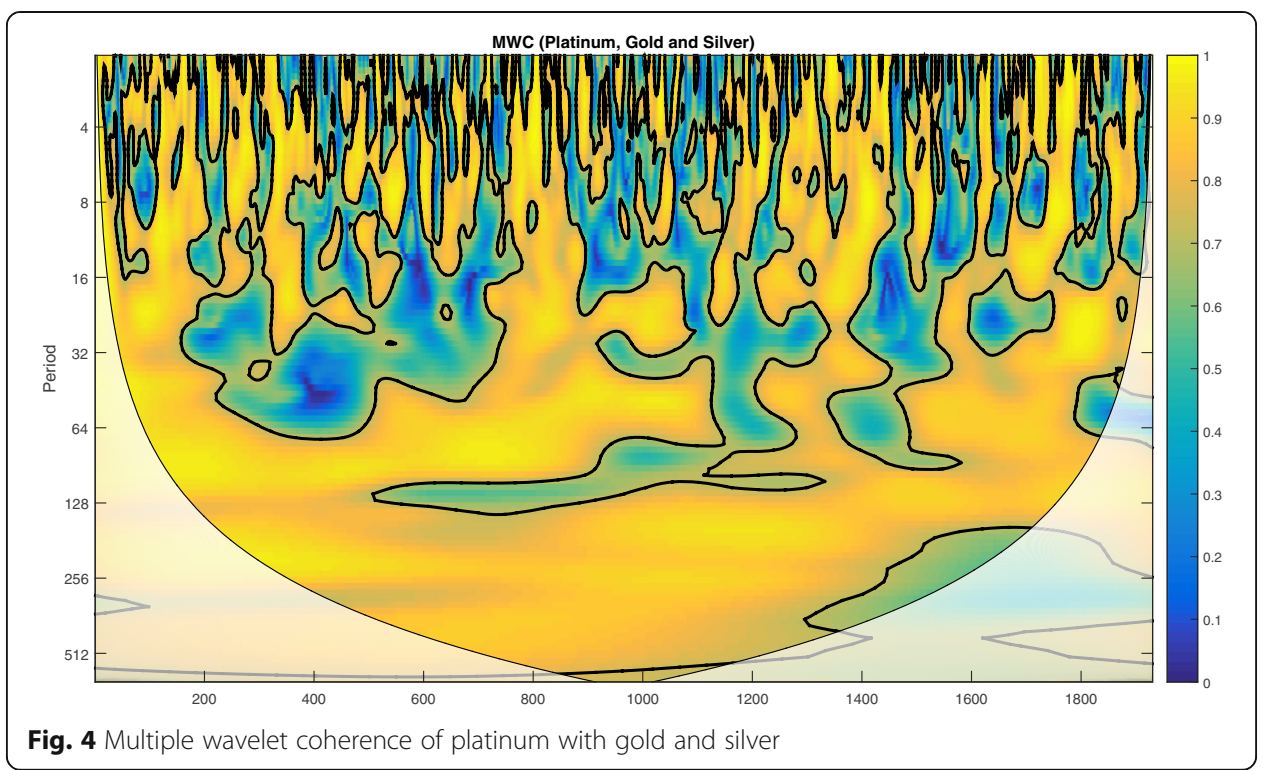

in-phase movement is well observed for platinum on 256-day period as shown in Fig. 4. This is found to be true for all metals at this scale. Hence we will be focusing on 256-day period for the rest of our study.

The generalized Hurst exponent considers the time series as a single time period and provides a single value that indicates the global behavior of the series. Hurst exponents define a continuum between a noise like time series (Hurst exponent between 0 and 1 ) and a random walk like time series (Hurst exponent above 1). The pink noise where Hurst exponent is equal to 1 separates between the noises $\mathrm{H}<1$ that have more apparent fast evolving fluctuations and random walks $\mathrm{H}>1$ that have more apparent slow evolving fluctuations (Ihlen, 2012). The validity of Hurst exponents may be established with the linear relationship for both the real data and the fluctuation function time series obtained at the specific scale as shown in Figs. 5 and 6. The log-log plots of other metals are shown in Appendix 2. A correlated (long range dependent) structure is observed when the Hurst exponent is in the interval of 0.5 and 1 . An anti-correlated (short range dependent) structure is observed when the Hurst exponent is in the interval of 0 and 0.5 (Ihlen, 2012). When Hurst exponent is higher than 1, the level of long-range dependency may not be expressed in terms of magnitude but one can easily conclude that it perpetuates its long-range dependency as strong as it protracts.

It is expected in this paper that the better performance of forecasting will be obtained due to the long range dependence and the multifractal behavior of the time series obtained. In Table 3, it is shown that the real time series data of all metals have generalized Hurst exponent even greater than 1, indicating a strong long range dependence.

When we calculated the local Hurst exponents at scale 256, the fluctuation function series obtained at the specified scale are still demonstrating multifractal behavior as strong as the raw data. There is only $10 \%$ difference for gold and silver and $2.7 \%$ for platinum. The main advantage of working with local Hurst exponents with respect to generalized Hurst exponent is the ability of local Hurst exponent to identify the time instant of structural changes within the time series (Ihlen, 2012). 


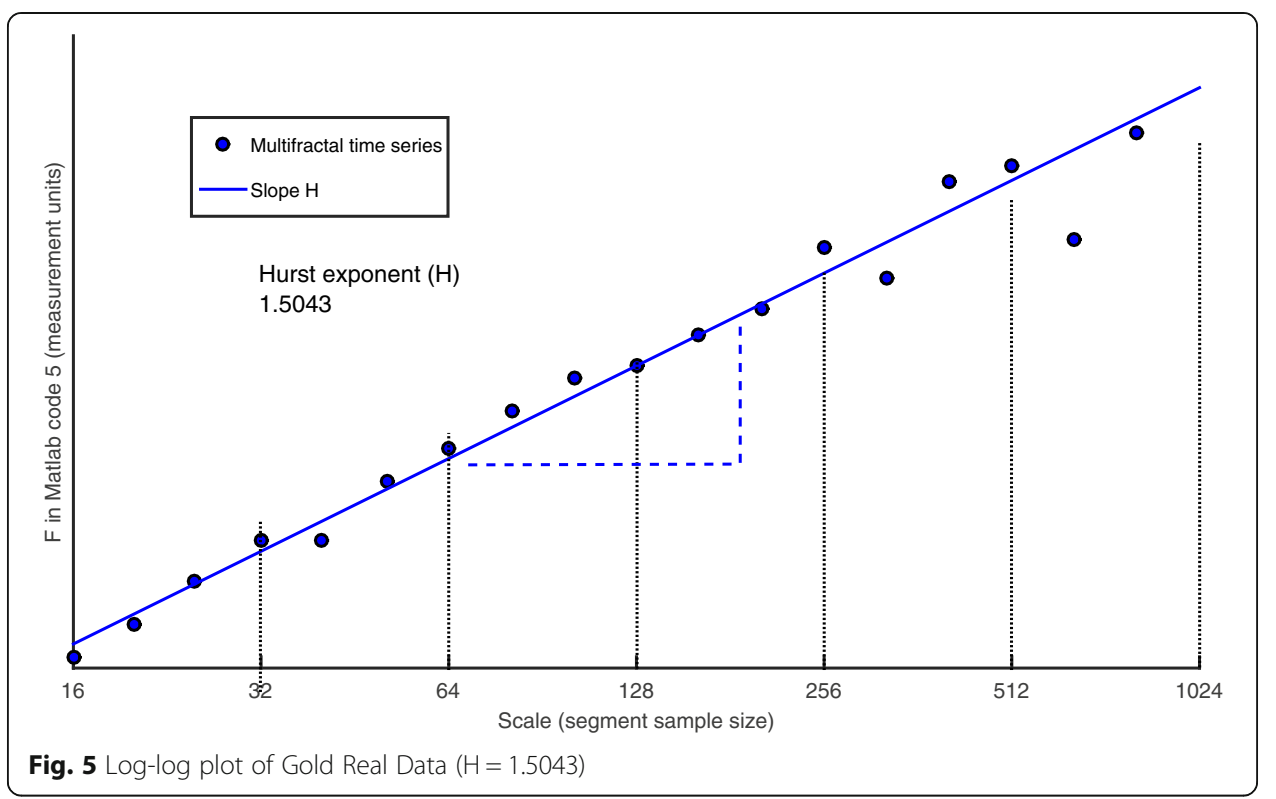

In Fig. 7, the real data of gold and its corresponding local Hurst exponents are displayed. The other metals may be found in Appendix 3. In Table 4, the minimum and maximum values of local Hurst exponents at scale 256 are listed. Even though gold has the lowest maximum value among all the metals, it does not have weakening long range dependence with the lowest Hurst exponent value of 1.03. Silver and platinum on the other hand have minimum Hurst exponents of 0.86 around the same time interval. This is indicating a weaker persistent behavior in terms of long range dependence. Silver and platinum are demonstrating stable long range dependence in the latest times of the series. However, as shown in Fig. 7, gold has a continuously increasing value of local Hurst exponents, suggesting stronger long range dependence with even smaller variations in price.

The local Hurst exponents are calculated by dividing the root mean square of residuals and log value of the corresponding scale for all periods in each scale one by one. It is our

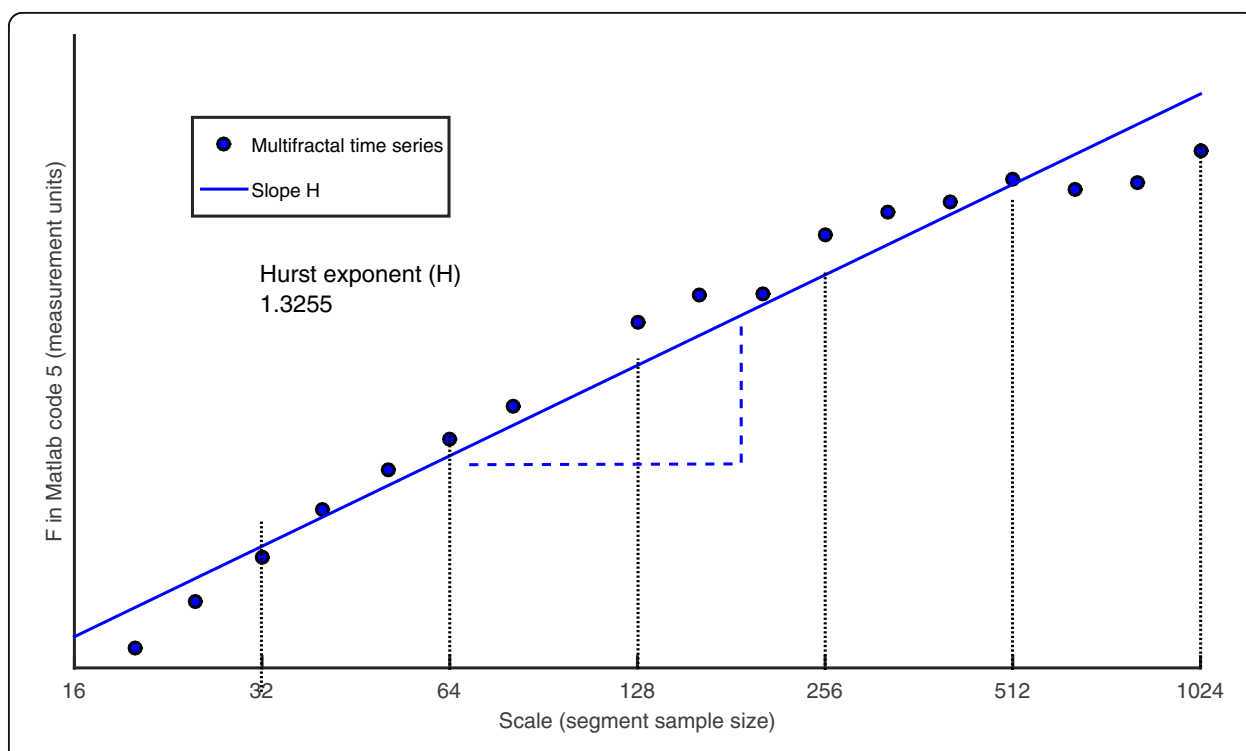

Fig. 6 Log-log plot of Gold at scale $256(H=1.3255)$ 
Table 3 Hurst Exponent of Real and Fluctuation function at scale 256

\begin{tabular}{llll}
\hline & Gold & Silver & Platinum \\
\hline General Hurst Exponents & 1.5043 & 1.4714 & 1.4927 \\
General Hurst Exponents of Fluctuation Function Time Series at Scale 256 & 1.3255 & 1.3237 & 1.4515 \\
$\%$ Change & $11.89 \%$ & $10.04 \%$ & $2.76 \%$ \\
\hline
\end{tabular}

assumption that this inter-calculated time series of root mean square of residuals during possess the features of the real data with stronger long-range dependency and may be used to estimate the shift of price change. In Fig. 8, this anew generated fluctuation function time series obtained during the calculation of local Hurst exponents at scale 256 are shown. These time series will be used to model and forecast the prices for the next 30 days. However due to the nature of the calculation, the values need to be rescaled with respect to the raw data. To do so, we calculated the ratio between the mean value of the fluctuation function and the mean value of the raw data. Then we divided the series with that ratio and obtained a new series closer to the real data in value.

Since we know that both the real data and the fluctuation function data exhibits multifractal behavior, autoregressive fractionally integrated moving average (ARFIMA) model with the real data and vector autoregressive fractionally integrated moving average (VARFIMA) model with the fluctuation function time series will be used to estimate a process and forecast for the next 30 days. Finally, we will compare the results accordingly.

\section{Forecasting results and discussion}

Multivariate models lead to more accurate results compared to scalar counter models (Tsay, 2013) because not only the historical data of each series but also other fractionally co-integrated variables in between the series are taken into consideration in VARFIMA models. The study of Das et al. in 2018 concludes that diversification benefits in pacific developed markets are limited due to higher degrees of integration (Das et al., 2018). We believe otherwise that it is important to set up highly correlated data set in order to establish efficiently estimated processes with better forecasting results. The raw data and fluctuation

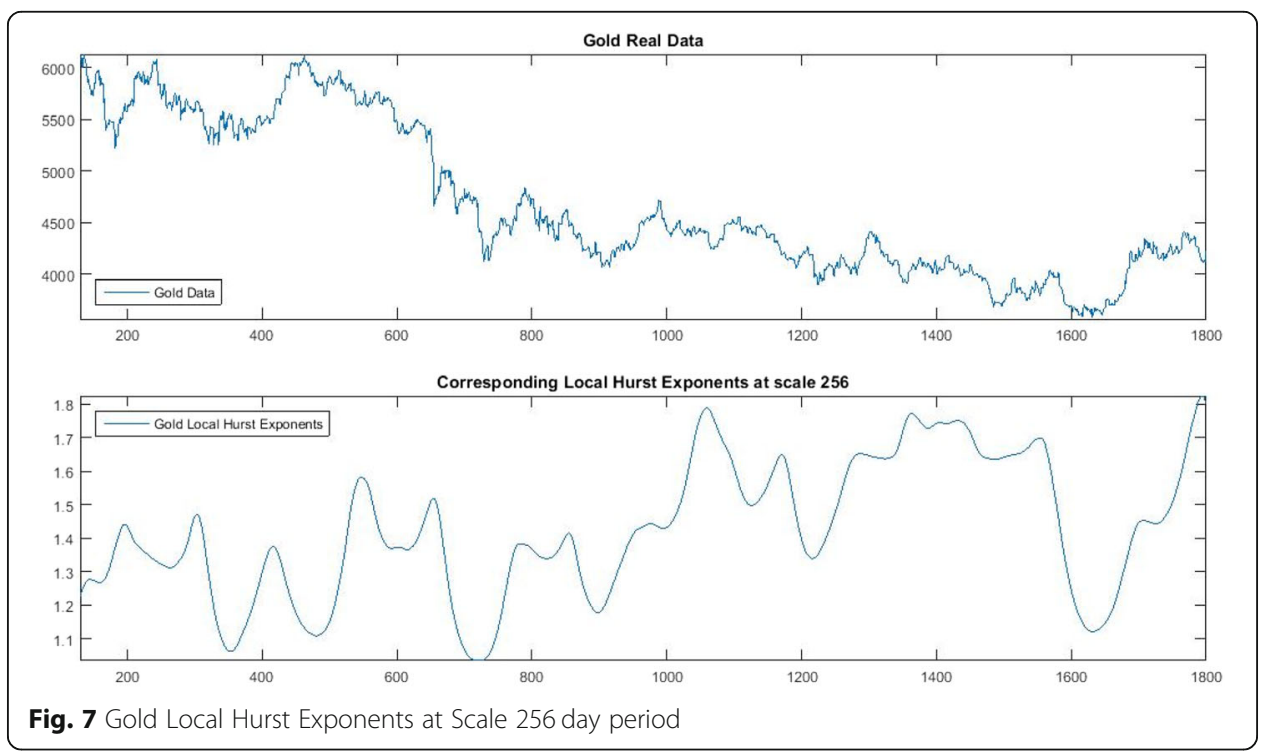


Table 4 Minimum and Maximum Values of Local Hurst exponents at scale 256

\begin{tabular}{llll}
\hline & Gold & Silver & Platinum \\
\hline Min Hloc & 1.0342 & 0.8648 & 0.8657 \\
Max Hloc & 1.8254 & 2.0615 & 2.133 \\
\hline
\end{tabular}

function time series data will be picked for the same interval for forecasting purposes. Raw data using ARFIMA process and fluctuation function data using 2D VARFIMA process will be forecasted and the results will be compared accordingly.

The reader must note that when 2D VARFIMA model are run, the forecasting results are provided with upper and lower limits of two dimensional vectors. In our results, we have eliminated the highest value of the upper boundaries and the lowest value of the lower boundaries displaying the narrowest band possible.

In Table 5, the dates of time series interval are given for each couple of metals. Once the time intervals with high coherence are determined (the yellow regions in Fig. 4), 6 time intervals with different length of time frames have randomly been chosen for forecasting purposes. Hence 12 forecasting trials have been demonstrated in total.

Forecasting for each metal has taken place for the same time interval in VARFIMA for the next 30 days. Figure 9 displays fraction function couple for gold and platinum from February 25th, 2011 to January 11th, 2012. The correlation of these series is approximately $80 \%$. VARFIMA model uses the same time series (gold with platinum and platinum with gold) to obtain the forecasting results of each metal and they are shown in Fig. 10 for gold and in Fig. 11 for platinum. As you can see from both of the results, forecasting with VARFIMA model have performed extremely well with respect to ARFIMA model of the raw data for the same time frame chosen.

This has been the case for all metals and time intervals chosen. ARFIMA (univariate model) have been used with the raw data itself and VARFIMA (multivariate model) have been used with the fluctuation function time series provided out of local Hurst exponent calculations.

We have seen that the upper and lower error bands of all forecasting trials using VARFIMA method have contained the actual value on the 30th day. Besides its observable ratification, the difference between the real value and the 30th day forecasting value were checked out to find out how well the performance of forecasting was and how close the

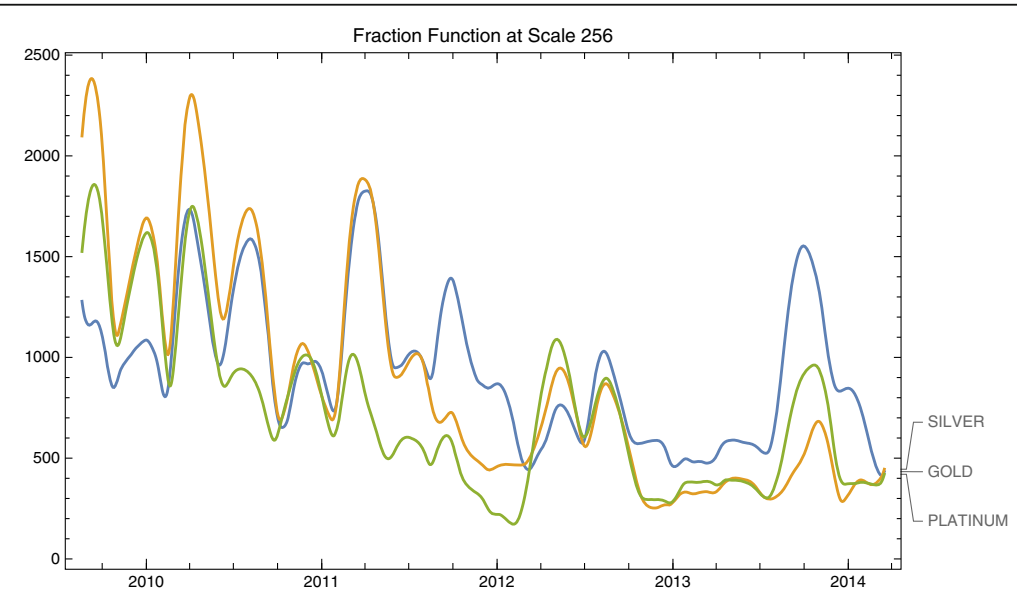

Fig. 8 Fraction function at scale 256 
Table 5 Dates and couples used to forecast precious metals

\begin{tabular}{llllll}
\hline & & $\#$ & From & To & Correlation \\
\hline Gold & Platinum & GP1 & 25.02 .2011 & 11.01 .2012 & 0.7905 \\
Gold & Platinum & GP2 & 19.12 .2011 & 18.04 .2012 & 0.6588 \\
Gold & Silver & GS1 & 25.02 .2011 & 06.01 .2012 & 0.8538 \\
Gold & Silver & GS2 & 06.03 .2010 & 18.05 .2012 & 0.8056 \\
Silver & Platinum & SP1 & 19.04 .2010 & 21.07 .2012 & 0.7189 \\
Silver & Platinum & SP2 & 26.02 .2011 & 11.01 .2012 & 0.8669 \\
\hline
\end{tabular}

results were for each trial. In seven out of 12 trials, the proximity to the actual value from VARFIMA trials were $10 \%$ or less of the difference value of ARFIMA results. In Table 6, the differences between 30th day forecasting value and real value are given for all forecasting results. In all trials, VARFIMA method has given closer values to the actual value. It is shown that VARFIMA has provided better forecasting results with respect to its counterpart which is in parallel with the findings of Pena and Sanchez (Peña \& Sanchez, 2007) that multivariate models perform better with respect to univariate models. Despite of the fact that some of the ARFIMA models have given smaller root mean square error values, they were abortive and have shown a decreasing trend in all of the results. On the other hand, the forecasting results with the use of VARFIMA model, the estimated function was able to follow an approximate path with the real data. It is also seen that the narrower range of prediction and lower root mean square error in the estimated models are achieved with the increased rate of correlation of the series.

Additionally, the present results may assist members of the financial world at varying degrees in their field. Vast number of the research papers report that the price of gold is an indicator of inflation (Ranson \& Wainright, 2005). This is because of commodity prices can respond to new information faster than any consumer prices (Mahdavi \& Zhou, 1997). Furthermore, Gold is treated as hedging instrument against inflation and exchange rates (Hammoudeh et al., 2010). Therefore, accurate forecasting (i.e. forecasts with smaller error bands) of the gold price will serve to monetary policymakers, hedge fund managers, international portfolio managers and central banks to make accurate investment decisions in the financial market. However, forecasting of gold prices is a formidable task. This is because of

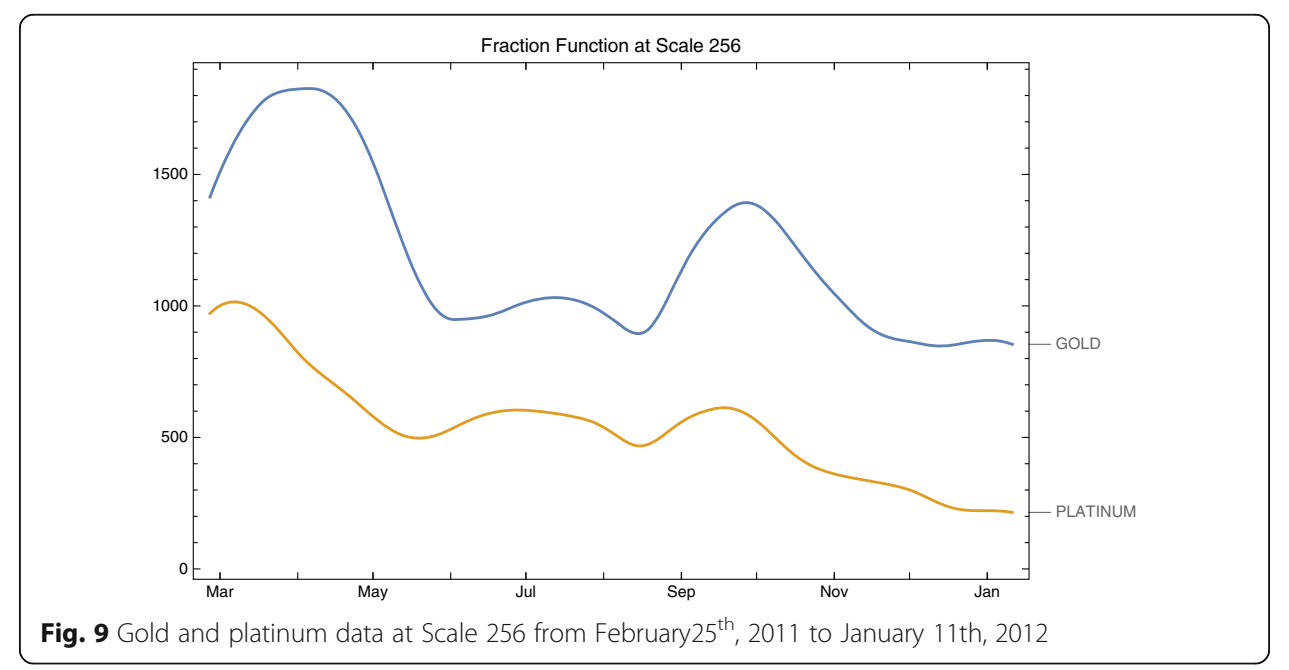




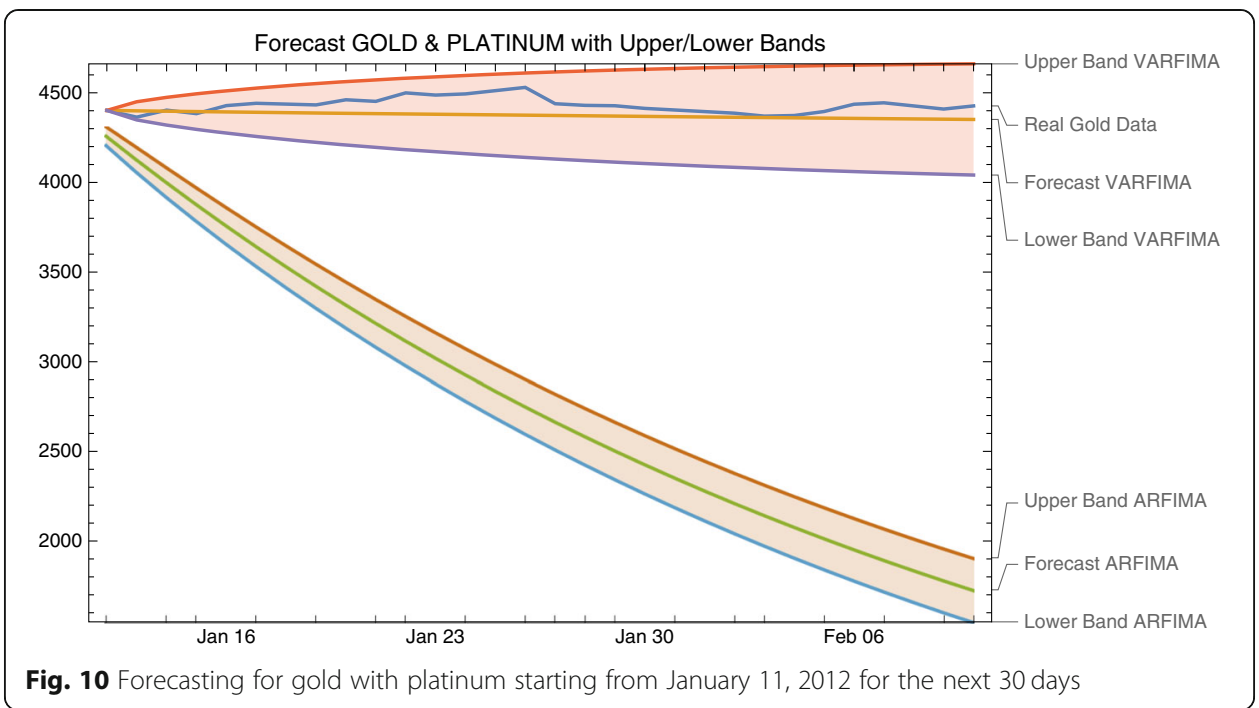

the multifractal nature of the gold prices. To the best of our knowledge there is no general approach for forecasting of multifractal data.

We have overcome this difficulty by determining the time scale in which gold, silver and platinum exhibit coherence for all times. This happens to be 256-day period. Furthermore, we fit VARFIMA model for this scale which leads to forecasting results with extremely narrow error bands. Although Silver and Platinum serve as coherent companions of Gold. We also have perfect forecasting performances since we consider them as a component of VARFIMA model. This will allow to make healthier assessment of inflation expectations. These empirical findings may particularly serve as decision aids for policy makers, hedge fund managers, international portfolio managers and gold exporters.

\section{Conclusion}

Multiple wavelet coherence and multifractal de-trended fluctuation analysis (two important tools in the analysis of financial time series) have been employed to explore the

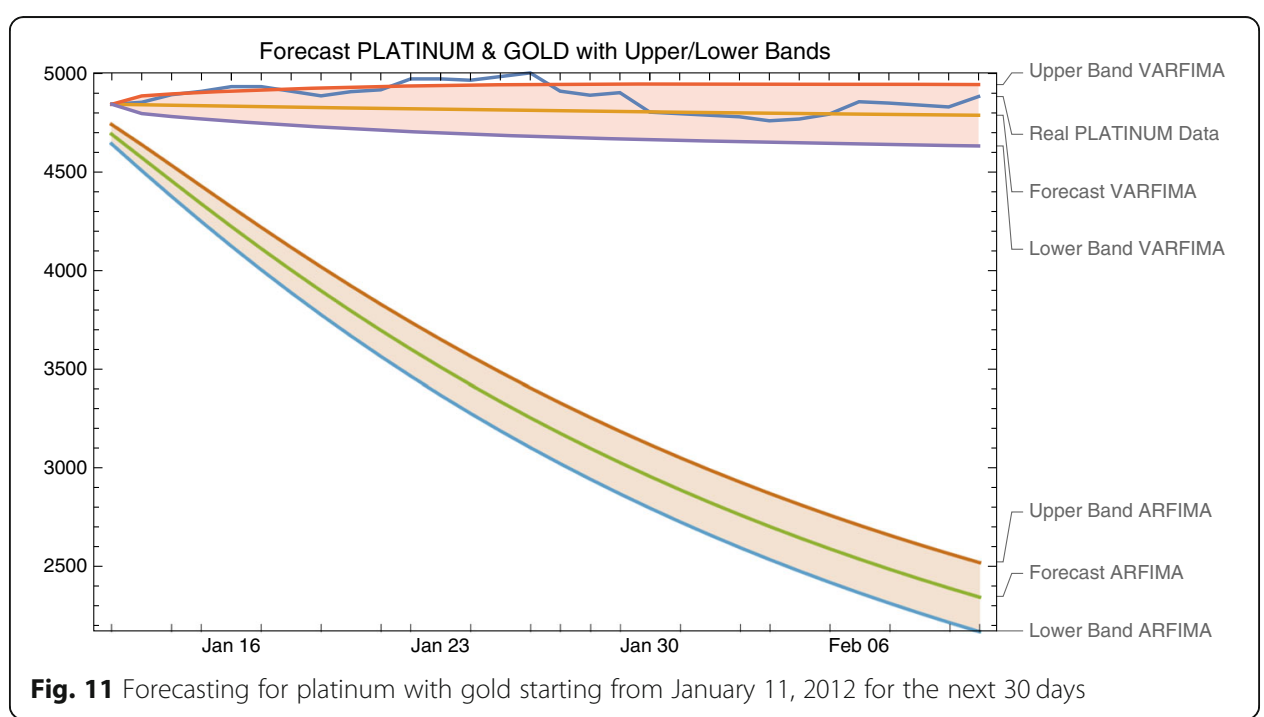


Table 6 The difference between 30th day forecast and real value

\begin{tabular}{lllllllll}
\hline & \multicolumn{7}{l}{ The Difference Between 30th Day Forecast \& Real Value } \\
\cline { 3 - 8 } & & ARFIMA & VARFIMA & $\%$ & & ARFIMA & VARFIMA & $\%$ \\
\hline GP1 & Gold & 2511 & 82 & 3.2 & Platinum & 2463 & 104 & 4.2 \\
GP2 & Gold & 1495 & 13 & 0.9 & Platinum & 2018 & 17 & 0.8 \\
GS1 & Gold & 2279 & 273 & 11.9 & Silver & 4836 & 29 & 0.6 \\
GS2 & Gold & 495 & 72 & 14.5 & Silver & 537 & 168 & 31.3 \\
SP1 & Silver & 814 & 585 & 71.8 & Platinum & 1105 & 106 & 9.9 \\
SP2 & Silver & 4254 & 561 & 13.2 & Platinum & 2467 & 63 & 2.5 \\
\hline
\end{tabular}

inter dependency and multifractality of precious metals, gold, silver and platinum. Firstly, it is presented that multiple wavelet coherence provides higher resolution to visualize in-phase movement of different time series in time and frequency space compared to any other traditional correlation function analysis (Yilmaz \& Unal, 2016). The direct observation allowed us to conclude that all three metals are highly correlated at higher periods throughout the time, especially around 256-day period.

We have observed that long range dependence is weaker over certain periods of time and frequency, which might be due to asymmetric error-correction mechanisms between precious metal prices during shocks or stresses. This could mean that it would take time at different rates for all metal prices to find equilibrium. This is parallel with the findings of (Kucher \& McCoskey, 2017) whom stated that the long run relationships between precious metals are strongly influenced by economic conditions.

Compared to univariate counterparts (ARFIMA), it is depicted that the consistency and performance of forecasting with multifractal time series is remarkably increased with multivariate models (VARFIMA) (Durr et al., 1997). This was also true in spite of the size of the data set chosen (Dueker \& Startz, 1998) where we have used set of data from 300 daily prices up to 1100 daily prices.

The generalized Hurst exponents of all precious metals have revealed that daily prices of precious metals do possess long range dependence with persistent structure and demonstrate multifractal behavior in general. Furthermore, local Hurst exponents of precious metals at 256-day period turned out to be multifractal as well. The local Hurst exponents are at the highest part of the scale during their latest timeframes. It indicates a strengthening trend in terms of long-range dependence with persistent structure (Ihlen, 2012), which means that the periods do not experience large variations and the prices are evolving in slower pace.

A new inter-calculated fluctuation function time series is generated during the calculations of local Hurst exponents. These fluctuation function time series are used to model and forecast the data for the next 30 days. Even though both of the methods (ARFIMA and VARFIMA) are adopted to integrate time series into the model fractionally and all series demonstrate multifractal behavior, ARFIMA model have almost never produced forecasting results as successful as VARFIMA model which have outperformed its univariate match conspicuously. This has proven the fact that obtaining data couples with higher correlation helps accomplish better forecasting results.

Jiang and Zhou developed multifractal detrended moving average cross correlation analysis (Jiang \& Zhou, 2011) based on detrended fluctuation analysis. For future research, it may be utilized to compare with the current methodology. 


\section{Appendix 1}

\section{Forecasting results}
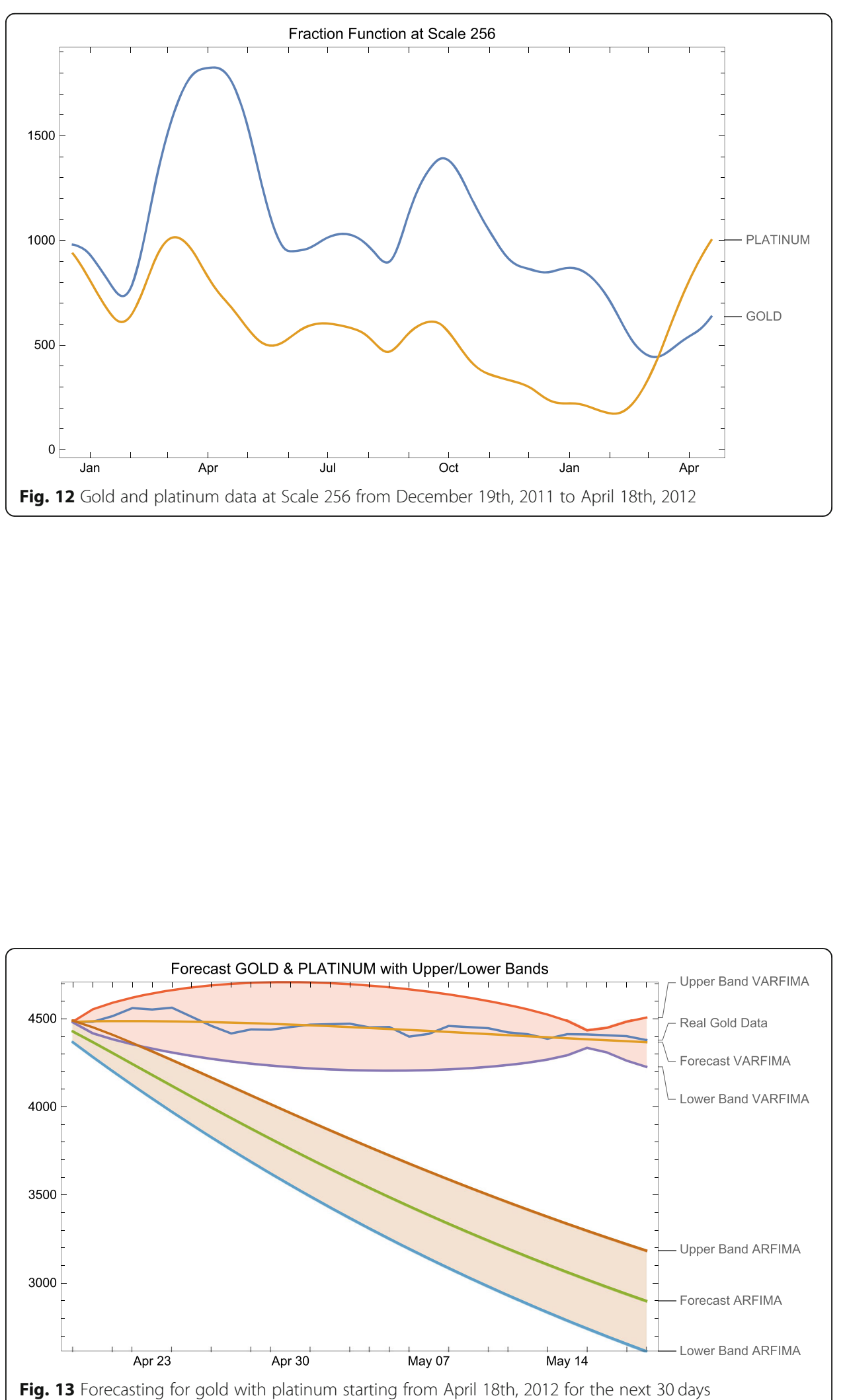

Fig. 13 Forecasting for gold with platinum starting from April 18th, 2012 for the next 30 days 

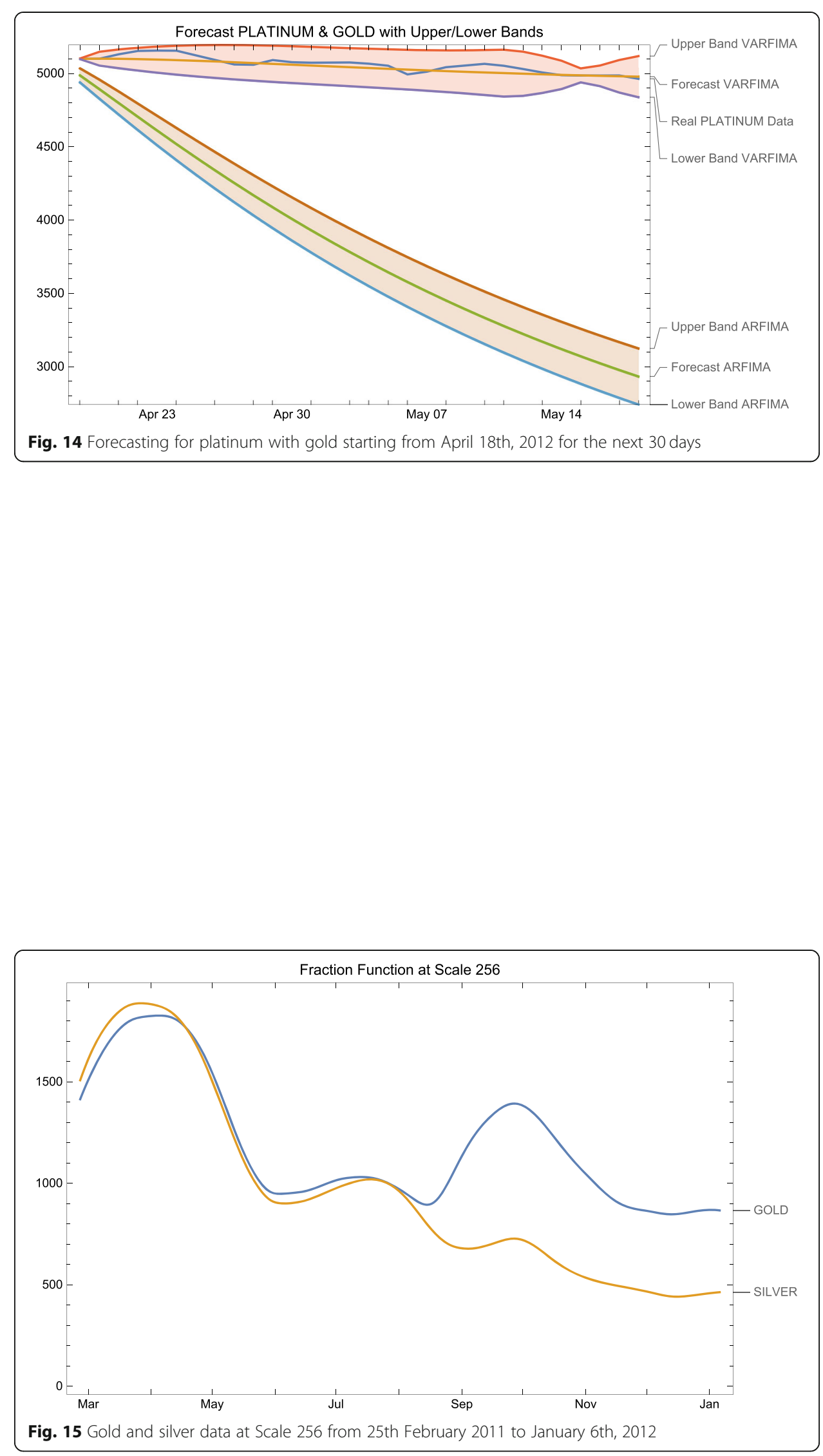

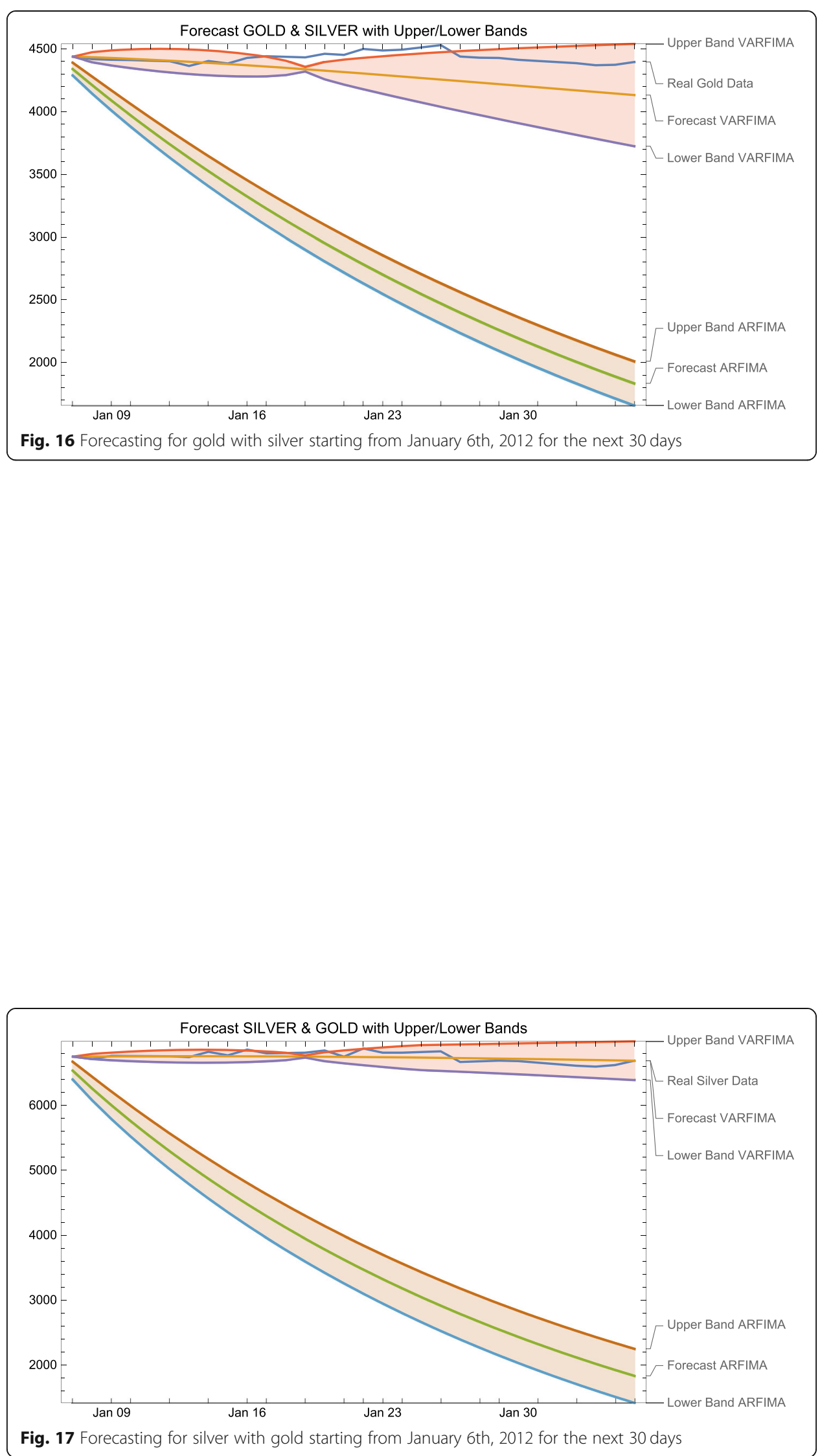


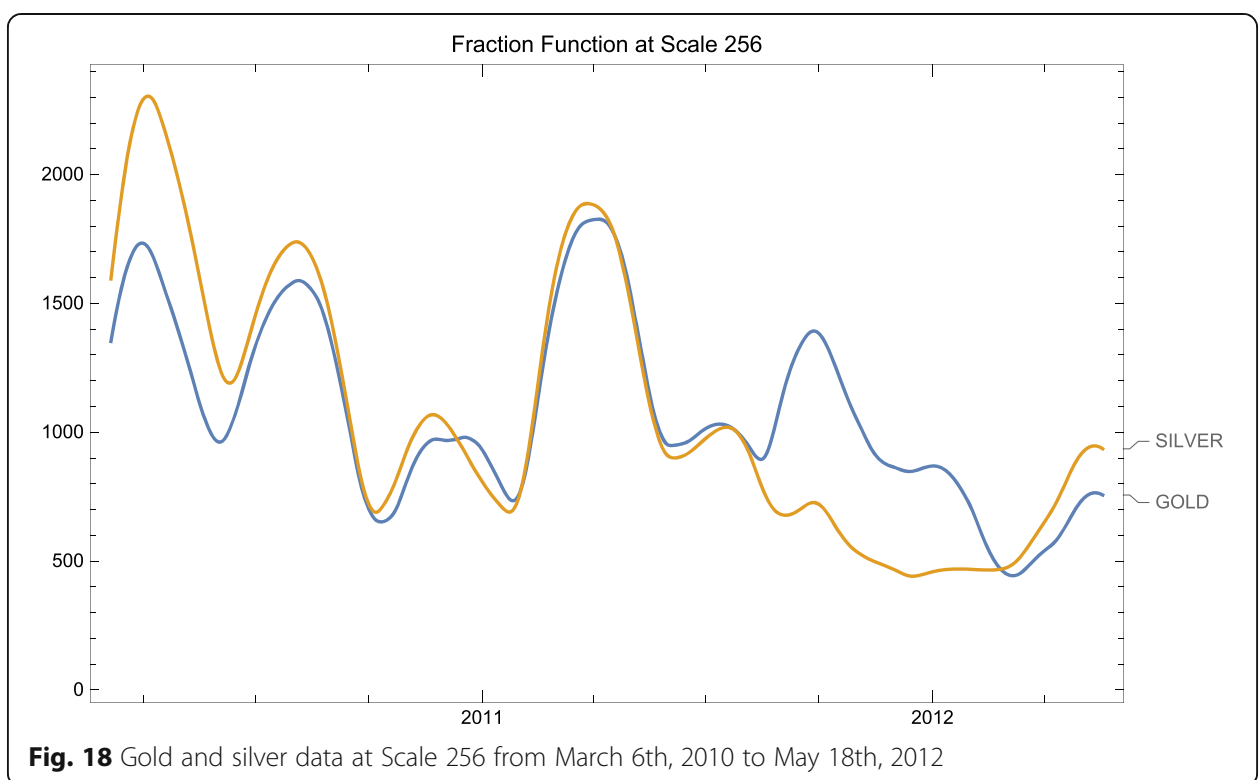

Fig. 18 Gold and silver data at Scale 256 from March 6th, 2010 to May 18th, 2012

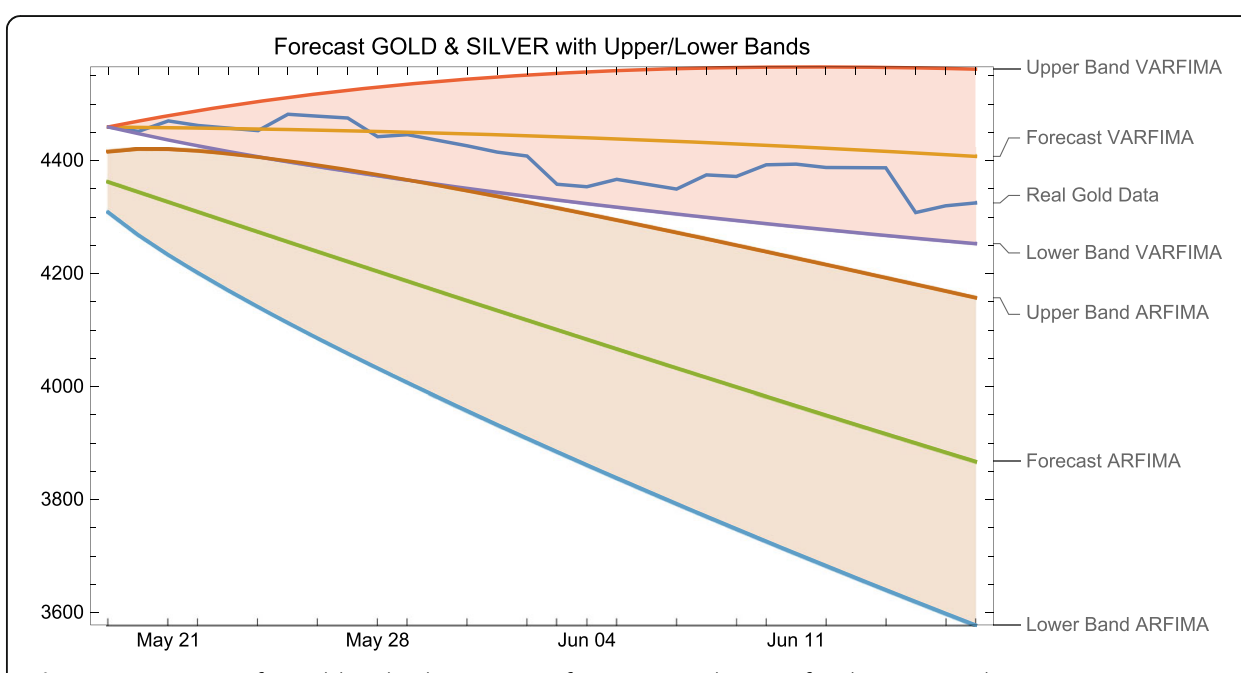

Fig. 19 Forecasting for gold with silver starting from May 18th, 2012 for the next 30 days 

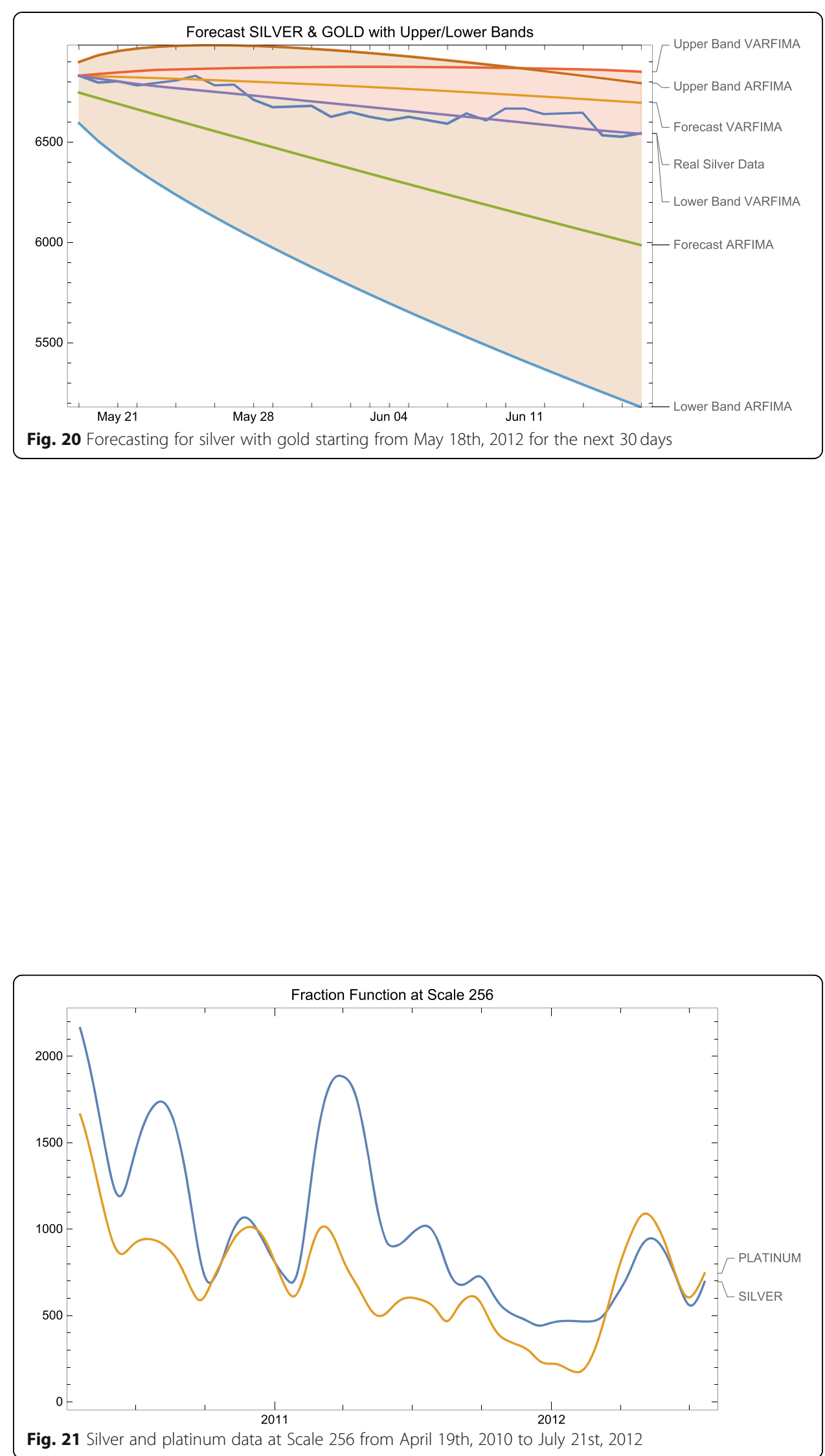

Fig. 21 Silver and platinum data at Scale 256 from April 19th, 2010 to July 21st, 2012 

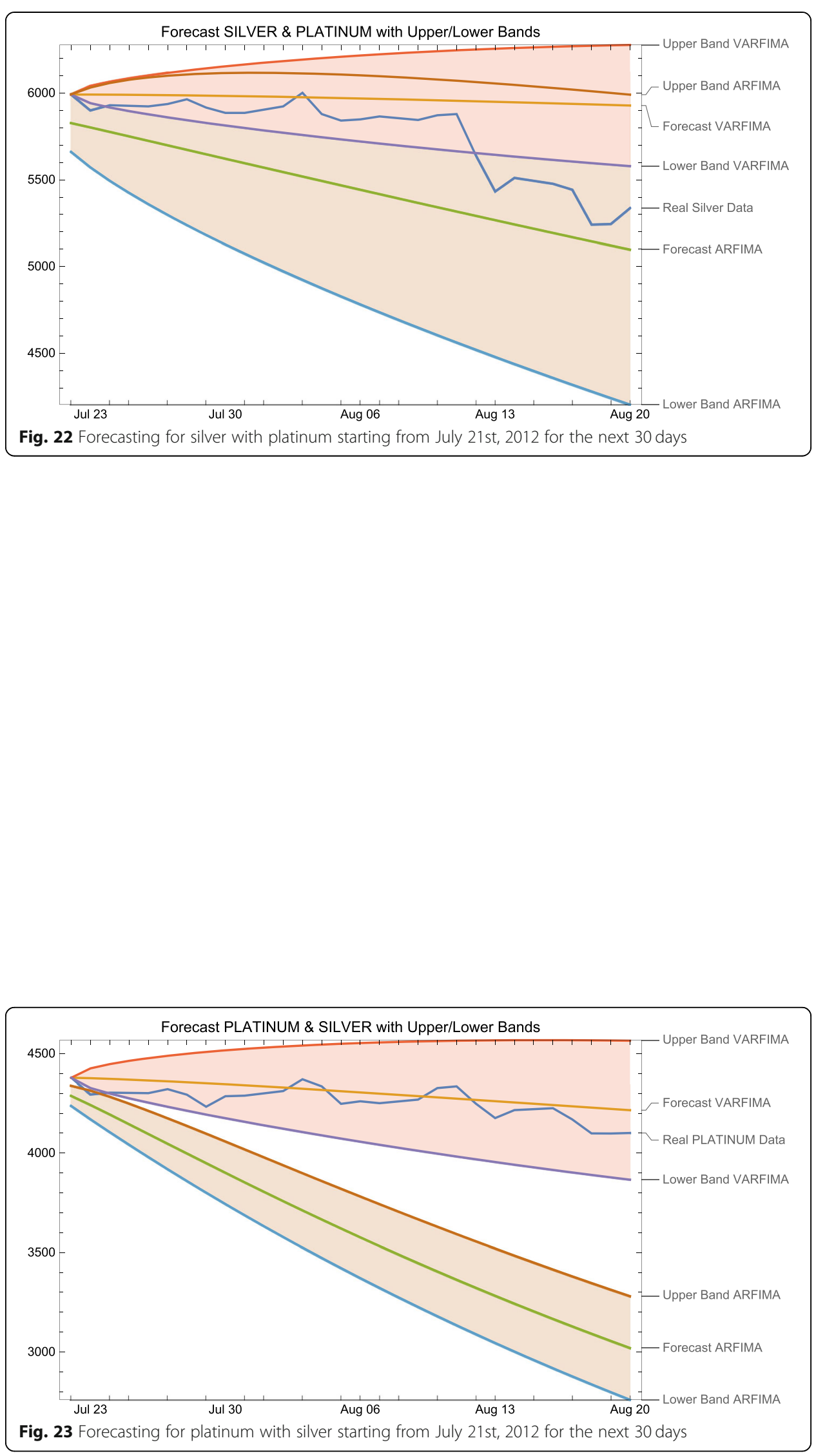


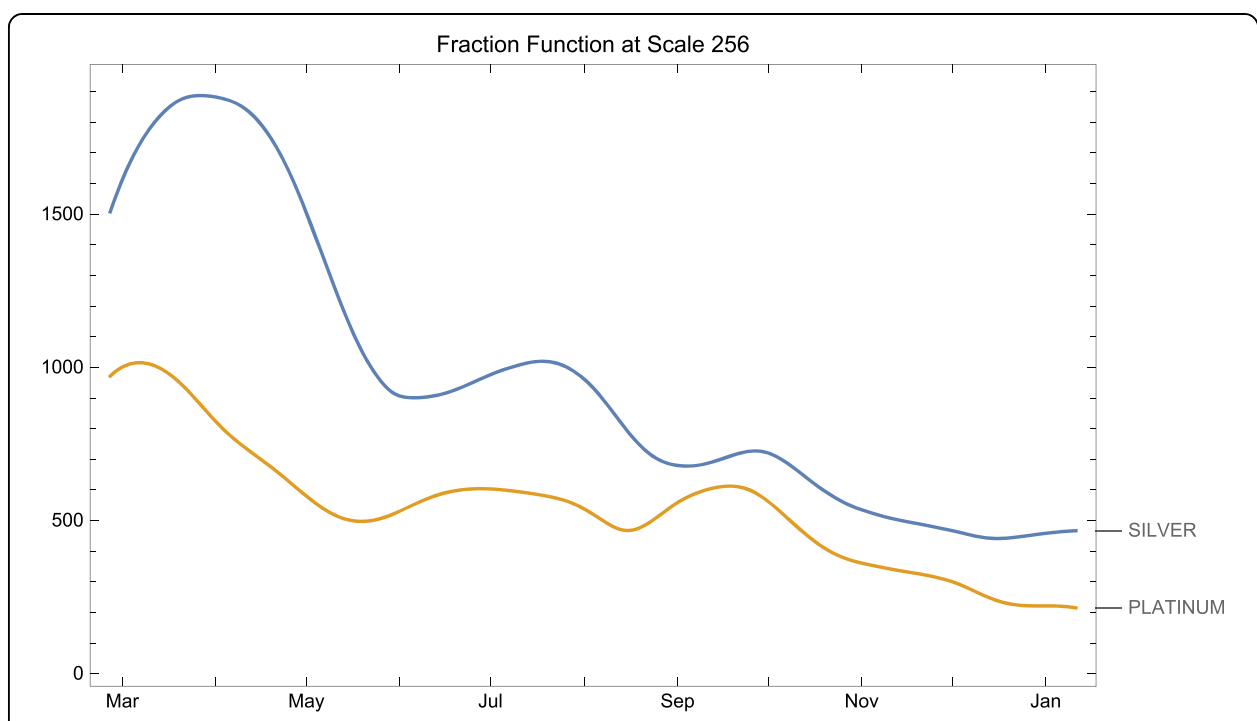

Fig. 24 Silver and platinum data at Scale 256 from February 25th, 2011 to January 11th, 2012

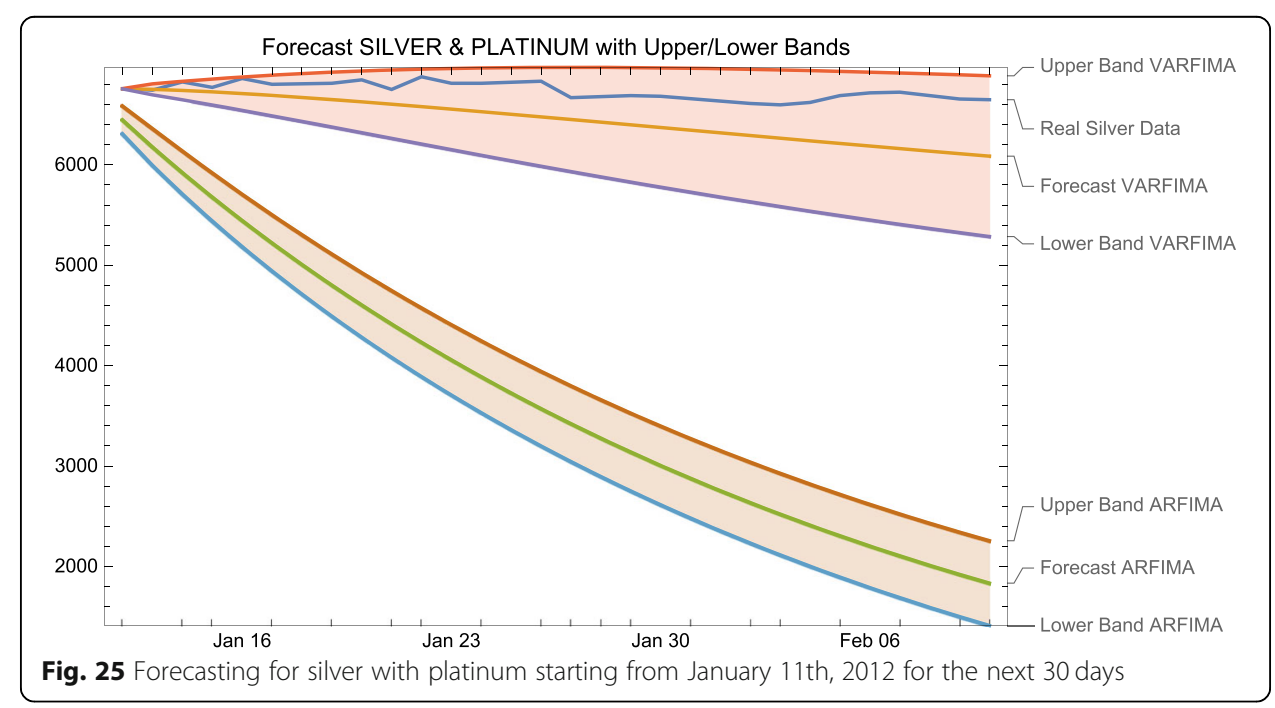




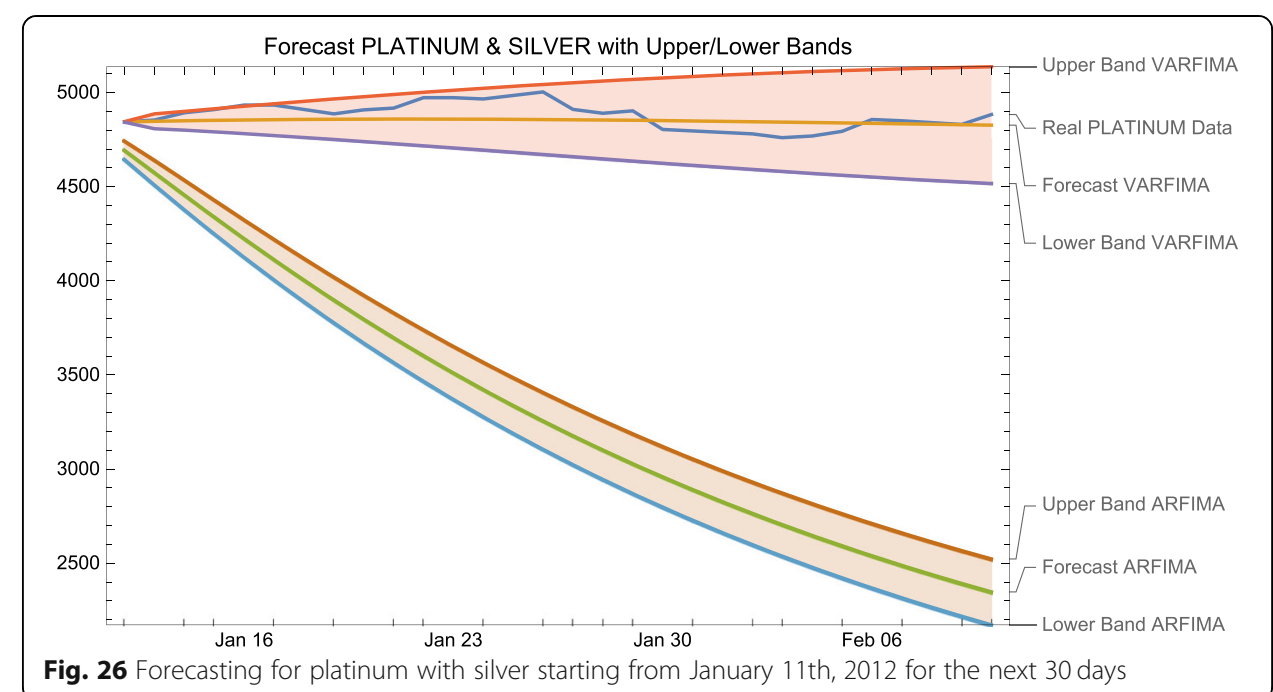

\section{Appendix 2}

Hurst exponents for real and scale 256 data

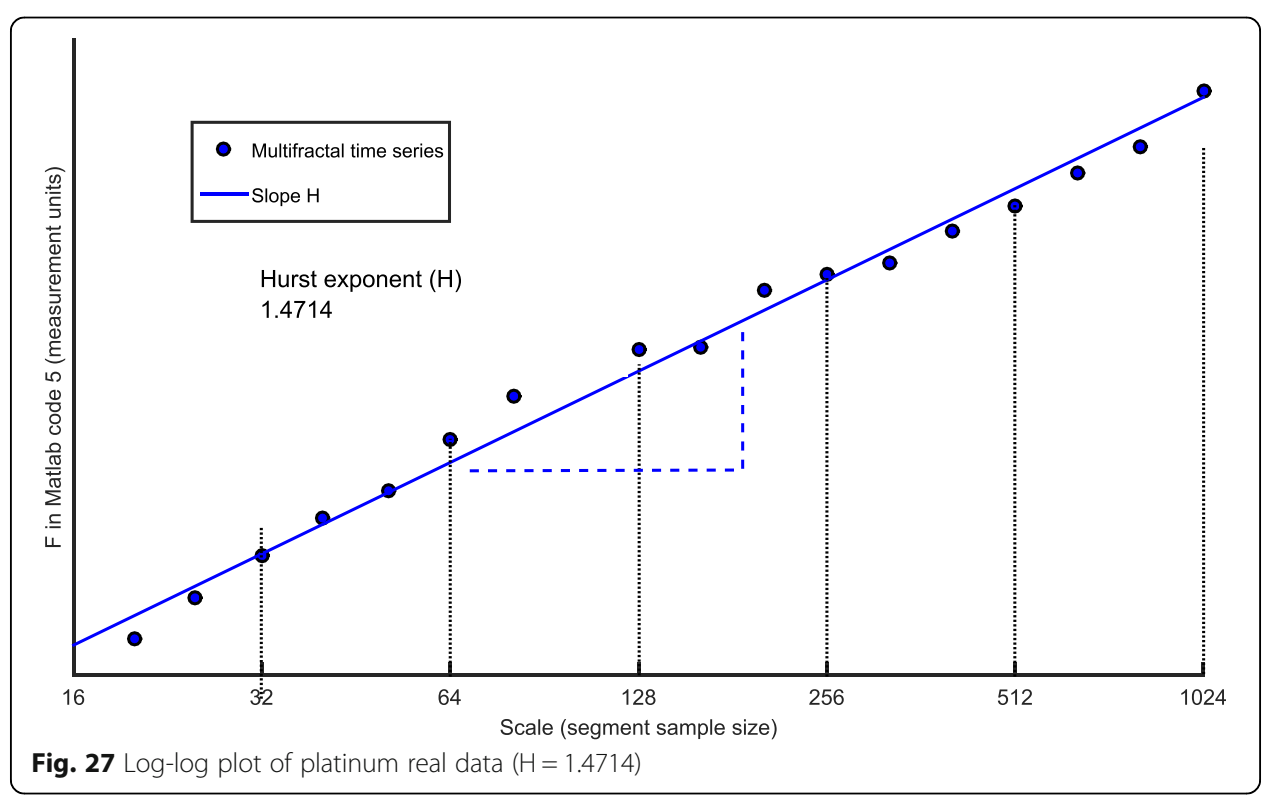



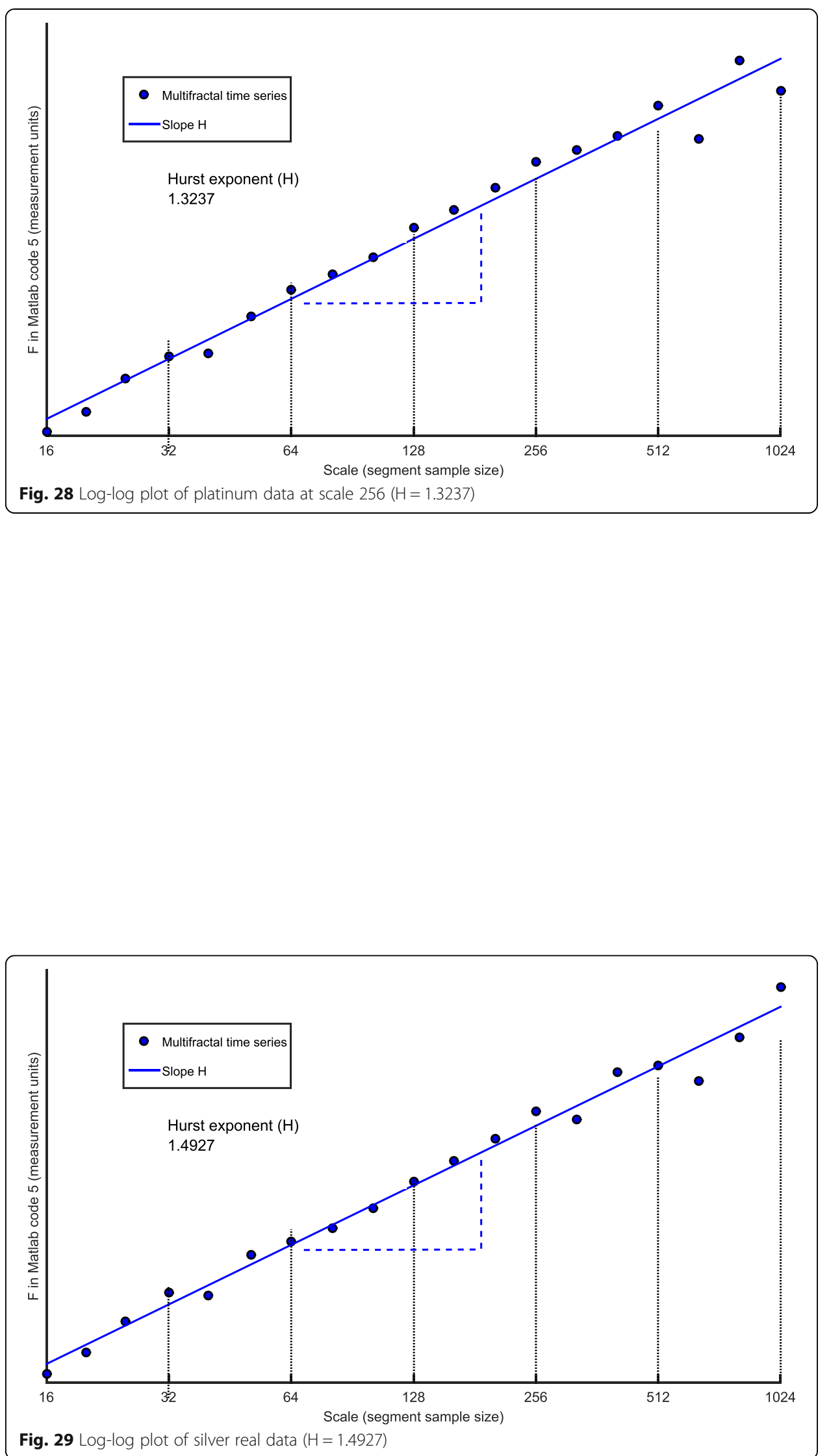

Fig. 29 Log-log plot of silver real data $(H=1.4927)$ 


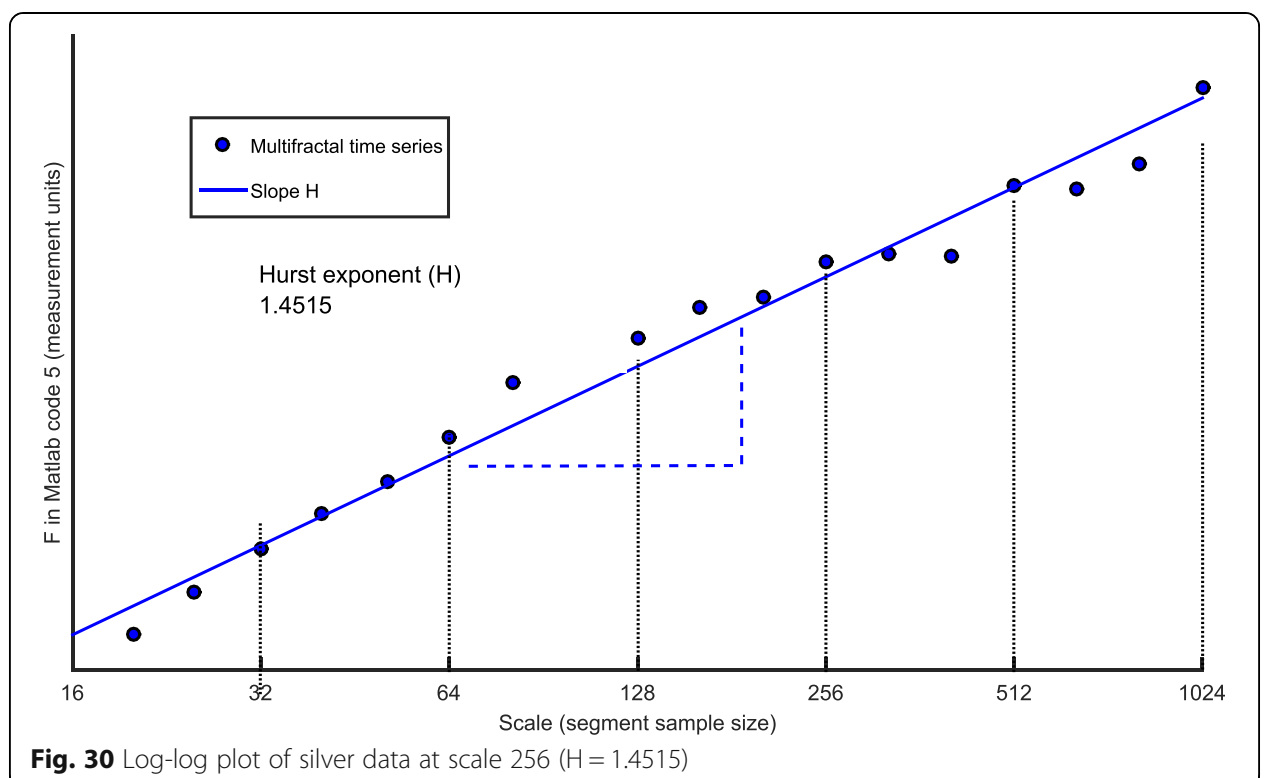

Fig. 30 Log-log plot of silver data at scale $256(H=1.4515)$

\section{Appendix 3}

\section{Local Hurst Exponents}

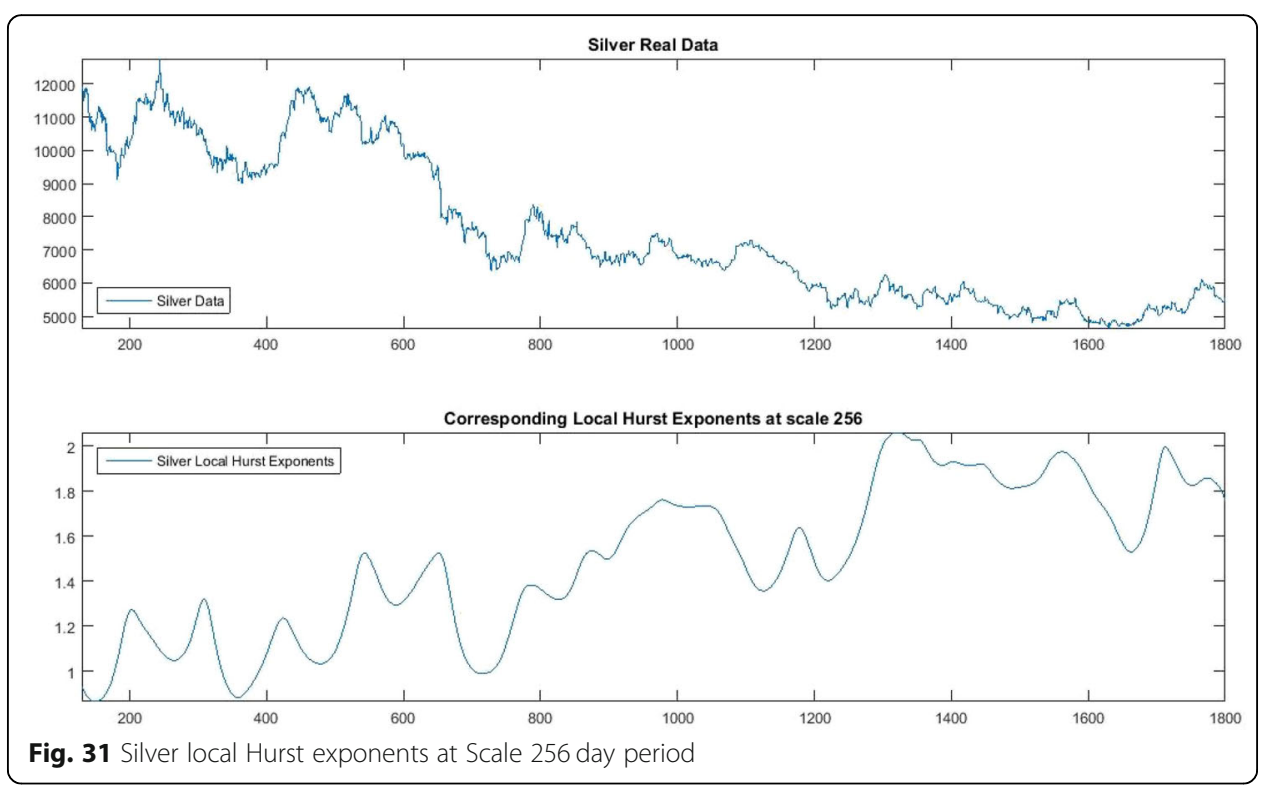




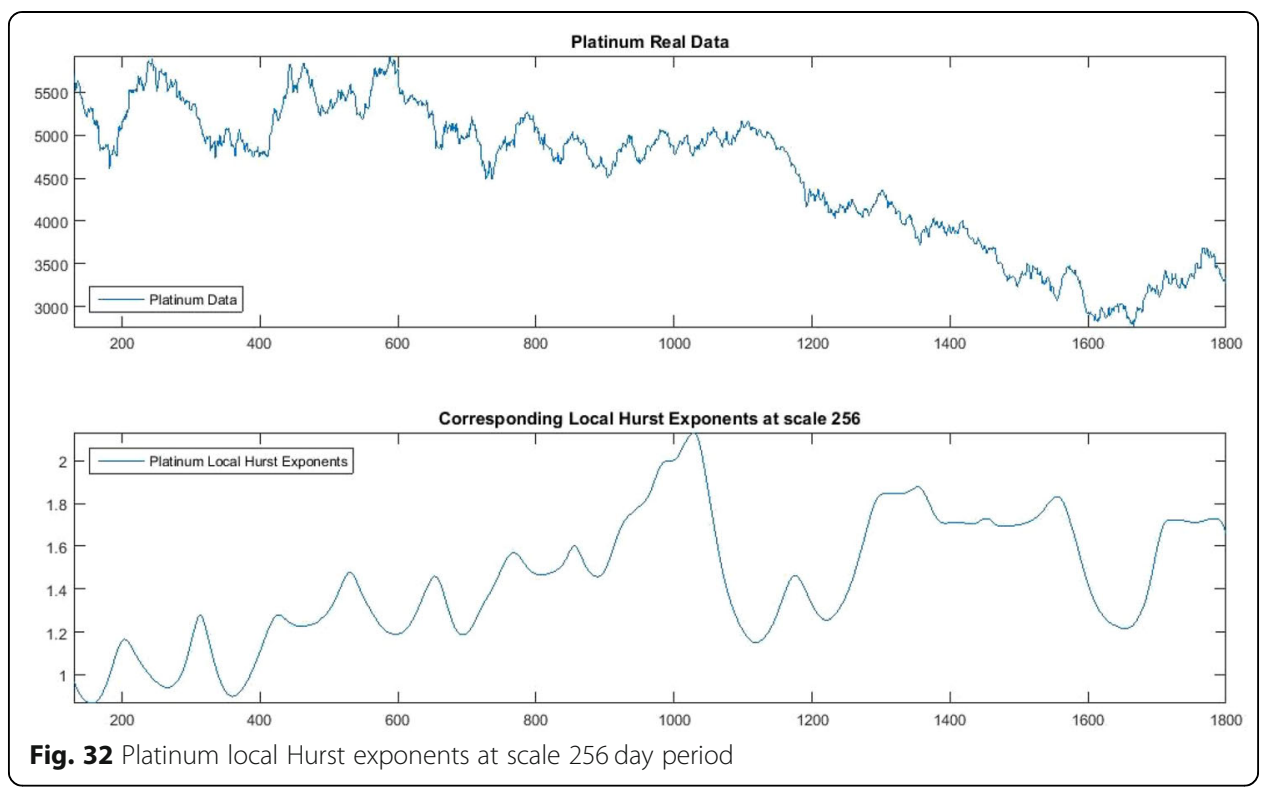

\section{Abbreviations}

ARFIMA: AutoRegressive Fractionally Integrated Moving Average; COI: Cone of Influence; CWT: Continuous Wavelet Transform; DWT: Discrete Wavelet Transform; MENA: Middle East and North African; MF-DFA: Multifractal De-Trended Fluctuation Analysis; MWC: Multiple Wavelet Coherence; VARFIMA: Vector AutoRegressvie Fractionally Integrated Moving Average

\section{Acknowledgements}

We would like to thank our anonymous referees for their constructive comments and valuable contributions.

\section{Funding}

Not Applicable.

\section{Availability of data and materials}

The datasets generated and/or analyzed during the current study are available from Yahoo Finances! or from corresponding author on reasonable request.

\section{Authors' contributions}

EO analyzed the data with multiple wavelet coherence and multifractal de-trended fluctuation analyses and generated forecasting results using vector autoregressive fractionally integrated moving average model. GU was the supervisor in construing the results, conclusion as well as in writing the manuscript. All authors read and approved the final manuscript. The content of the manuscript has not been published or submitted for publication elsewhere.

\section{Competing interests}

The authors declare that they have no competing interests.

\section{Publisher's Note}

Springer Nature remains neutral with regard to jurisdictional claims in published maps and institutional affiliations.

\section{Author details}

${ }^{1}$ Faculty of Economy and Administrative Sciences, Istanbul Aydin University, Istanbul, Turkey. ${ }^{2}$ Faculty of Economics, Administrative and Social Sciences, Bahcesehir University, Istanbul, Turkey.

Received: 23 November 2018 Accepted: 15 April 2019

Published online: 12 June 2019

\section{References}

Aguiar-Conraria L, Soares MJ (2012) The continuous wavelet transform: a primer. Working paper no. 16/2011. NIPE

- University of Minho, Portugal, pp 1-43

Aguiar-Conraria L, Soares MJ (2013) The continuous wavelet transform: moving beyond uni- and bivariate analysis. J Econ Surv 28(2):344-375

Akaike H (1974) Stochastic theory of minimal realization. IEEE Trans. Autom. Control 19(6):667-674 
J. Barunik, E Kocenda and L Vacha, Gold, oil, and stocks, (Preprint, 2013), arXiv: 1308. 0210 [q-fin.ST]

Benbachir S, Alaoui MH (2011) A multifractal detrended fluctuation analysis of the Moroccan Dirham with respect to the US dollar. Int Econ Finance J 6(2):287-300

Box-Steiensmeier JM, Tomlinson AR (2000) Fractional integration methods in political science. Elect. Stud. 19(1):63-76

Burrus CS, Gopinath RA, Guo H (1998) Introduction to wavelets and wavelet transforms - a primer. Prentice Hall, New Jersey Clarke HD, Lebo M (2003) Fractional (co) integration and government party support in Britain. Br. J. Polit. Sci. 33(2):283-301

Das D, Bhowmik P, Jana RK (2018) A multiscale analysis of stock return co-movement and spillovers: evidence from Pacific developed markets. Physica A 502:379-393

Das D, Kannadhasan M, Al-Yahyaee KH, Yoon S (2017) A wavelet analysis of co-movements in Asian gold markets. Physica A 492:192-206

Dick OE, Mochovikova IA (2011) Multifractal and wavelet analysis of epileptic seizures. Chaos Theory:159-166

Dueker M, Startz R (1998) Maximum-likelihood estimation of fractionally cointegration with an application to U.S. and Canadian bond rates. Rev. Econ. Stat. 80(3):420-426

Dunsmuir WTM, Hannan EJ (1976) Vector linear time series models. Adv. Appl. Probab 8(2):339-364

Durr RH, Gilmour JB, Wolbrecht C (1997) Explaining congressional approval. Am. J. Polit. Sci. 41(1):175-207

Gencay R (2002) Discrete wavelet transforms, An introduction to wavelets and other filtering methods. Finance Econ 1:96160

Grinsted J, Moore C, Jevrejeva S (2004) Application of the cross wavelet transform and wavelet coherence to geophysical time series. Nonlinear Process Geophys 11(5/6):561566

Gülerce M, Ünal G (2016) Using wavelet analysis to uncover the co-movement behavior of multiple energy commodity prices. Int. J. Wavelets Multiresolution Inf. Process 14(6):1650047

Hammoudeh S, Yuan Y, McAleer M, Thompson M (2010) Precious metals-exchange rate volatility transmission and hedging strategies. Int Rev Econ Financ 19(4):698-710

Hannan EJ (1981) Estimating the dimension of a linear system. J. Multivar. Anal 11(4):459-473

Haven E (2012) De-noising option prices with the wavelet method. Eur J Oper Res 222(1):104-112

He K, Chen Y, Tso GKF (2017) Price forecasting in the precious metal market: a multivariate EMD denoising approach. Recources Policy 54:9-24

Ihlen E (2012) Introduction to multifractal detrended fluctuation analysis in Matlab. Front Physiol 3(141):1-18

Jiang Z, Zhou W (2011) Multifractal detrending moving-average cross correlation analysis. Phys Rev E 81(1):1-11

Kantelhardt JW, Zschiegner SA, Koscielny-Bunde E, Havlin S, Bunde A, Stanley HE (2002) Multifractal Detrended Fluctuation Analysis of Nonstationary Time Series. Physica A 316:87-114

Klein T (2017) Dynamic correlation of precious metals and flight-to-quality in developed markets. Financ Res Lett 23:283-290

Kucher O, McCoskey S (2017) The long-run relationship between precious metal prices and the business cycle. Q. Rev. Econ. Finance 65(C):263-275

Mahdavi S, Zhou S (1997) Gold and commodity prices as leading indicators of inflation: tests of long-run relationship and predictive performance. J Econ Bus 49(5):475-489

Mandelbrot BB, Van Ness JW (1968) Fractional Brownian motion, fractional noises and its applications. SIAM Rev 10(4):254282

McCarthy J, Orlov a G (2012) Time-frequency analysis of crude oil and S\&P 500 futures contracts. Quant. Finan 12(12):1893-1908

Oral E, Unal G (2017a) Co-movement of precious metals and forecasting using scale by scale wavelet transform. Int J Financial Eng 4(1):1-21 1750007

Oral E, Unal G (2017b) Dynamic correlation of eastern and western markets and forecasting: Scale-by-scale wavelet-based approach. Int J Financial Eng 4(3):1-22 1750040

Peña D, Sanchez I (2007) Measuring the advantages of multivariate vs univariate forecasts. J. Time Ser. Anal. 28(6):886-909

Quenouille MH (1957) The analysis of multiple time series. Grien, London

Ranson, D., Wainright, H.C., Why gold, not oil, is the superior predictor of inflation. Gold report, World Gold Council, November, 2005

Reboredo JC, Rivera-Castro MA (2014) Gold and exchange rates: downside risk and hedging at different investment horizons. Int Review Econ Finance 34:267-279

Sensoy A (2013) Generalized Hurst exponent approach to efficiency in MENA markets. Physica A 392:5019-5026

Sensoy A, Tabak BM (2015) Time-varying long memory in European Union stock markets. Physica A 436:147-158

Sowell F (1989) Maximum likelihood estimation of fractionally integrated time series models working paper. CarnegieMellon University

Thompson JR, Wilson JR (2016) Multifractal detrended fluctuation analysis: practical applications to financial time series. Math Comput Simul 126(C):63-88

Tiwari AK, Albulescu CT, Yoon S-M (2017) A multifractal detrended fluctuation analysis of financial market efficiency: comparison using Dow Jones sector ETF indices. Physica A 483:182-192

Torrence AC, Compo GP (1998) A practical guide to wavelet analysis. Bull. Am. Meteorol. Soc. 79(1):61-78

Tsay RS (2013) Multivariate time series analysis: with R and financial applications, Wiley Senes in probability and statistics. Wiley, New York

Yilmaz A, Unal G (2016) Co-movement analysis of Asian stock markets against FTSE100 and S\&P 500: wavelet based approach. Int J Financial Eng 3(4):1-19 1650033

Zhang X, Zeng M, Meng Q (2017) Asymmetric multiscale multifractal analysis of wind speed signals. Int J Modern Physics C 28(11):1750137

Zhu H, Zhang W (2017) Multifractal property of Chinese stock market in CSI 800 index based on MF-DFA approach. Physica A 490:497-503 